This is the author's final version of the Topical Review published in IOP Nanotechnology on 22 July, 2014.

It should be cited as R.R. Hartmann, J. Kono and M.E. Portnoi, Nanotechnology 25, 32201 (2014).

The published version can be accessed at http://iopscience.iop.org/0957-4484/25/32/322001

\title{
Terahertz Science and Technology of Carbon Nanomaterials
}

\author{
R. R. Hartmann ${ }^{1}$, J. Kono ${ }^{2}$, and M. E. Portnoi ${ }^{3}$ \\ ${ }^{1}$ Physics Department, De La Salle University, 2401 Taft Avenue, Manila, Philippines \\ ${ }^{2}$ Department of Electrical and Computer Engineering and Department of Physics \\ and Astronomy, Rice University, 6100 Main St., MS-378, Houston, Texas 77005, USA \\ ${ }^{3}$ School of Physics, University of Exeter, Stocker Road, Exeter EX4 4QL, UK and \\ International Institute of Physics, Universidade Federal do Rio Grande do Norte, \\ Natal - RN, Brazil \\ E-mail: Richard.Hartmann@dlsu.edu.ph, kono@rice.edu, \\ M.E.Portnoi@exeter.ac.uk
}

\begin{abstract}
The diverse applications of terahertz radiation and its importance to fundamental science makes finding ways to generate, manipulate, and detect terahertz radiation one of the key areas of modern applied physics. One approach is to utilize carbon nanomaterials, in particular, single-wall carbon nanotubes and graphene. Their novel optical and electronic properties offer much promise to the field of terahertz science and technology. This article describes the past, current, and future of the terahertz science and technology of carbon nanotubes and graphene. We will review fundamental studies such as terahertz dynamic conductivity, terahertz nonlinearities, and ultrafast carrier dynamics as well as terahertz applications such as terahertz sources, detectors, modulators, antennas, and polarizers.
\end{abstract}




\section{Introduction}

The last three decades have witnessed explosive progress in the research and technology of carbon-based nanostructures highlighted by the discovery of fullerenes [1], rediscovery in the 1980s of the detonation technique of producing nanodiamonds [2] which resulted in a dramatic increase in the studies of their properties and applications [3], syntheses of carbon nanotubes [4] and the unprecedented success of a simple scotch tape exfoliation technique [5] leading to the spectacular rise of graphene [6]. The same period has seen wide-range efforts in bridging the so-called terahertz ( $\mathrm{THz})$ gap.

$\mathrm{THz}$ radiation lies between its better studied counterparts: microwave and infrared radiation in the electromagnetic spectrum. In this frequency range, electronic transport and optical phenomena merge with one another, and classical waves (in the microwave region) make the transition to quantum mechanical photons (in the optical regime). Therefore, understanding $\mathrm{THz}$ phenomena requires a multi-perspective approach. In condensed matter, many elementary and collective low energy excitations occur in the $\mathrm{THz}$ range such as plasmons, magnons, and superconducting energy gaps, and the majority of dynamical phenomena in solids such as scattering, tunneling, and recombination occur on characteristic times scales of picoseconds, i.e., in the $\mathrm{THz}$ frequency range.

Filling the $\mathrm{THz}$ gap is a challenging area in modern device physics [7], for this region of the electromagnetic spectrum presents difficulties in both generating coherent sources and creating sensitive detectors. However, the rewards of exploiting this gap are great, owing to the diverse applications of $\mathrm{THz}$ radiation. For example, the vibrational breathing modes of many large molecules occur in the $\mathrm{THz}$ domain making $\mathrm{THz}$ spectroscopy a potentially powerful tool for the identification and characterization of biomolecules [8-12]. Furthermore, the non-ionizing nature of $\mathrm{THz}$ radiation means that it is seen by many as the future of imaging technology, and it also has promising applications in biomedicine and biosensing. As well as utilizing $\mathrm{THz}$ technology for pharmaceutical research [13] and biomedical diagnostic devices, other potential uses range from security applications such as the sensing and detection of biological hazards and explosives [7], through to communication technology and astrophysics.

In the last decade, there has been significant progress made in the development of ultrafast laser based $\mathrm{THz}$ sources [14], quantum cascade [15] and free electron lasers [16], which operate in the $\mathrm{THz}$ range, as well as synchrotron-based $\mathrm{THz}$ sources [17]. However, current $\mathrm{THz}$ sources and detectors often suffer from low output power, are often considerable in size, and operate at liquid helium temperatures. For this reason, the search for ultra bright, coherent, and compact $\mathrm{THz}$ sources and detectors is one of the key areas of modern applied physics [18]. The unique position of the $\mathrm{THz}$ range, in the gap between the parts of electromagnetic spectrum that are accessible by either electronic or optical devices, leads to an unprecedented diversity in approaches to bridging this gap [7,19-27]. One approach is to utilize carbon nanomaterials, in particular, graphene and its one dimensional counterpart, the carbon nanotube [23], 

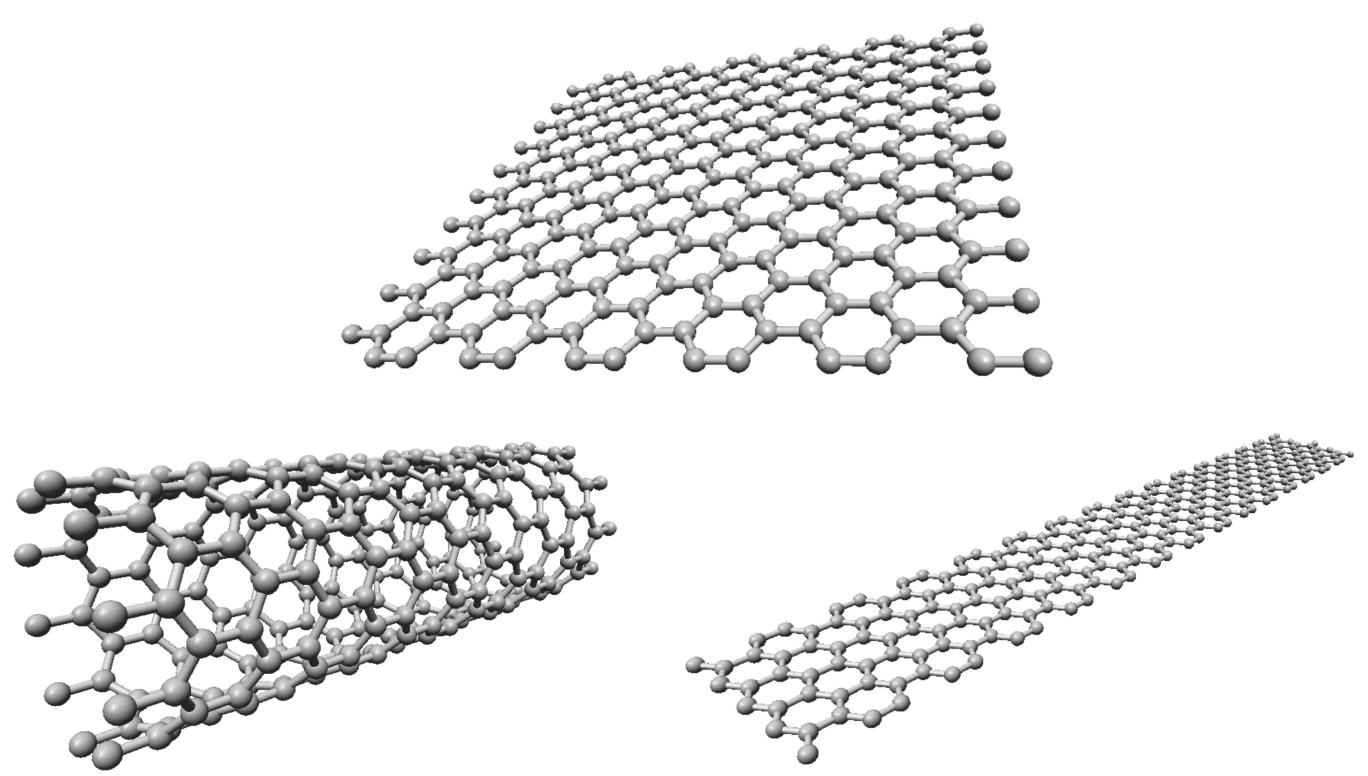

Figure 1. Graphene (top) is formed by carbon atoms arranged in a two-dimensional honeycomb crystal lattice. Graphene can be rolled into a cylinder to form carbon nanotubes (bottom left) or cut into graphene nanoribbons (bottom right).

whose optical and electronic properties are as interesting and diverse as their potential applications.

\subsection{What are carbon nanomaterials?}

Carbon's ability to exist in many different forms is due to the fact that carbon's four valence electrons may hybridize in many ways. This hybridization may be $\mathrm{sp}, \mathrm{sp}^{2}$ or $\mathrm{sp}^{3}$, allowing carbon to form linear chains, planar sheets and tetrahedral structures. $\mathrm{sp}^{2}$-bonded carbon can form a honeycomb crystal lattice, one atom thick, known as graphene. This one atom thick sheet of carbon is the building block of many carbon nanomaterials (see figure 1). Graphene can be rolled into a seamless cylinder to form carbon nanotubes, cut into graphene nanoribbons or fashioned into buckyballs. These low dimensional forms of nanocarbon could serve as the building blocks of carbon-based optoelectronic devices of the future.

1.1.1. Graphene Graphene's carbon atom's $2 s, 2 p_{x}$ and $2 p_{y}$ orbitals form $\sigma$ bonds to their nearest neighbors, and these bonds determine the crystal's structural properties. The remaining $2 p_{z}$ orbitals hybridize to form weaker, more delocalized $\pi$ bonds, and these dictate the optical and transport properties of the material. In general, the electronic structure of carbon nanomaterials can be described using a simple-tight binding model [28] of graphene's $\pi$-electrons. Unlike conventional systems, whose charge carriers are described by the Schrödinger equation with an effective mass, graphene's charge carriers are described by the same equation used to describe two-dimensional 


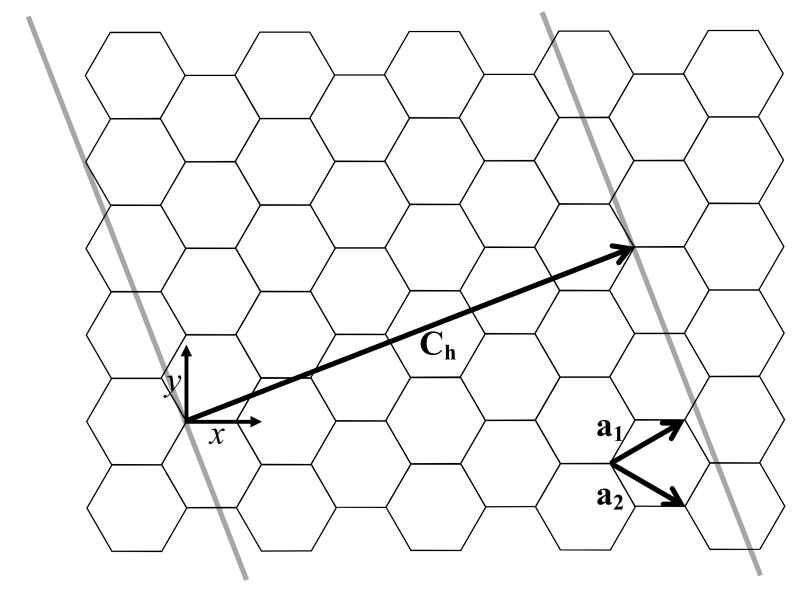

Figure 2. An unrolled carbon nanotube. The cylinder is formed by rolling the graphene sheet along the chiral vector, $\mathbf{C}_{\mathbf{h}}$, such that its start and end points coincide. $\mathbf{a}_{1}$ and $\mathbf{a}_{\mathbf{2}}$ are the graphene primitive lattice vectors.

massless Dirac fermions, the Dirac-Weyl equation [29-31]. The consequence of this unusual spectrum is that many effects that were once the in realms of high-energy particle physics are now measurable in a solid state system [32,33]. Graphene exhibits unusual mechanical, thermal and, most of all, electronic properties [31], which allows the observation of interesting effects such as the suppression of backscattering [34], an unconventional quantum Hall effect $[30,35]$, and a range of effects based on the Klein paradox [32]. The existence of massless Dirac fermions has been confirmed by magnetotransport measurements such as the integer quantum Hall effect [30,35], and the linear spectrum results in an optical conductance defined by the fine structure constant [36-38]. The relativistic nature of graphene's charge carriers makes confinement difficult owing to the fact that particles do not experience back scattering in a smooth electrostatic potential $[32,34]$. However, lateral confinement can be achieved by cutting graphene into nanoribbons, therefore allowing graphene to be used as a switching device. The geometrical confinement effects in graphene nanoribbons are similar to carbon nanotubes, but the boundary conditions imposed on the wavefunction due to edge type can lead to drastically different electronic properties [39-41] such as the existence of edge states.

1.1.2. Carbon Nanotubes A single-walled carbon nanotube (SWCNT) is formed by rolling a graphene sheet into a seamless cylinder $[28,42-44]$. The way in which the nanotube is rolled is described by the chiral vector $\mathbf{C}_{\mathbf{h}}$, which connects crystallographically equivalent sites of the unrolled graphene lattice (see figure 2). The chiral vector, is defined via the graphene primitive lattice vectors, $\mathbf{a}_{\mathbf{1}}$ and $\mathbf{a}_{\mathbf{2}}$, as $\mathbf{C}_{\mathbf{h}}(n, m)=n \mathbf{a}_{\mathbf{1}}+m \mathbf{a}_{\mathbf{2}} \equiv(n, m)$, where $n$ and $m$ are integers. If $n=m$ the nanotubes are called armchair nanotubes, and when $m=0$ the nanotubes are referred to as zigzag nanotubes; all other cases are classified as chiral nanotubes. For the case of $n-m=3 p$, 
where $p$ is a non-zero integer, the tubes are known as quasi-metallic tubes [45].

The manner in which the tube is rolled strongly dictates its electronic properties: the SWCNT can either be metallic, a narrow-gap semiconductor, or semiconducting. Within the frame of a simple zone-folding model of the $\pi$-electron graphene spectrum, armchair and quasi-metallic carbon nanotubes can be considered as one-dimensional analogs of graphene, since in this model the electron low-energy spectrum is linearly dependent on the electron wavevector. The electron energy spectrum, $\varepsilon(k)$, of the aforementioned SWCNTs is given by $\varepsilon(k)= \pm \hbar v_{\mathrm{F}}|k|$, where $k$ is measured from where the conduction and valence bands coincide and $v_{\mathrm{F}} \approx 9.8 \times 10^{5} \mathrm{~m} / \mathrm{s}$ is the Fermi velocity of graphene. However, due to curvature effects quasi-metallic SWCNTs are in fact narrowgap semiconductors [45], their bandgap given by $\varepsilon_{g}=\hbar v_{F} a_{\mathrm{C}-\mathrm{C}} \cos 3 \theta /\left(8 R^{2}\right)[46,47]$, where $a_{\mathrm{C}-\mathrm{C}}=1.42 \AA$ is the nearest-neighbor distance between two carbon atoms, $R$ is the nanotube radius, and $\theta=\arctan [\sqrt{3} m /(2 n+m)]$ is the chiral angle [28]. Their bandgap can be in the range of $\mathrm{THz}$ photon energies.

\subsection{Why are they good for THz science and technology?}

While initial investigations on these materials concentrated on DC characteristics, recent theoretical studies have instigated a flurry of new experimental activities to uncover unusual AC properties. Both nanotubes and graphene are expected to show exotic $\mathrm{THz}$ dynamics that can lead to innovative optoelectronic applications [48-52]. These properties are inherently related to their unique, low-dimensional band structure, combined with many-body interactions of quantum-confined carriers. In the presence of external magnetic fields and electric fields, certain types of nanotube develop strong $\mathrm{THz}$ optical transitions, giving rise to the possibility of utilizing them as highly tunable, optically-active materials in $\mathrm{THz}$ devices [49,53-58], and their desirable electronic properties and highly anisotropic optical properties make them ideally suited for $\mathrm{THz}$ antenna and polarizer applications [59-65]. Nanotubes also hold the promise of ballistic $\mathrm{THz}$ transistors [66] that could supersede traditional silicon technology.

As a gapless semiconductor with ultra high carrier mobility, graphene is a natural material for THz applications, and the ability to modify graphene's charge carrier density through electronic gating makes graphene ideally suited for optoelectronic applications. Its charge carriers can be further manipulated by externally applied magnetic fields, allowing tunable broadband detectors to be realized [67]. Graphene is also predicted to be a gain medium for $\mathrm{THz}$ lasers [68] which can operate at room temperature, an exciting prospect considering the dearth of $\mathrm{THz}$ sources. Equally exciting is graphene's ability to support surface plasmon modes in the THz regime [69-71], which can be tuned by electronic gating, therefore paving the way for novel graphene-based plasmonic devices that operate in the $\mathrm{THz}$ regime. 


\section{THz Science and Technology of Carbon Nanotubes}

\subsection{Dynamic Conductivity}

For one-dimensional (1-D) electron systems, including SWCNTs, there have been theoretical calculations of $\sigma(\omega)$, taking into account interactions and disorder to varying degrees (see, e.g., [72-77] and pp. 219-237 of [78]). Specifically for a metallic SWCNT, Ando [74] calculated $\sigma(\omega)$ within a self-consistent Born approximation, which indicated that there can exist non-Drude-like conductivity, depending on the range of scattering potentials.

In a 1-D conductor, electron-electron interactions lead to a breakdown of the Fermi liquid model. In this instance, the charge carriers are described as a Tomonaga-Luttinger liquid $[79,80]$. Tomonaga-Luttinger liquids were studied both theoretically $[81,82]$ and experimentally in single [83-86] and multi-wall nanotubes [87,88]. Burke showed theoretically that these 1-D plasmon modes can be excited at gigahertz frequencies [75]. However, it is currently unclear whether or not the single-particle or collective excitations dominate the high frequency response of carbon nanotubes [89,90].

A number of experimental $\mathrm{THz} /$ far-infrared spectroscopic studies have been performed over the last decade on SWCNTs of various forms [59-62, 64, 65, 91-98], producing an array of conflicting results with contradicting interpretations. This is partly due to the widely differing types of samples used in these studies - grown by different methods (HiPco, CoMoCAT, CVD, Arc Discharge, and Laser Ablation) and put in a variety of polymer films that are transparent in the $\mathrm{THz}$ range. Nanotubes in most of these samples were bundled and typically consisted of a mixture of semiconducting and metallic nanotubes with a wide distribution of diameters. One common spectral feature that many groups have detected is a broad absorption peak around $4 \mathrm{THz}$ (or $135 \mathrm{~cm}^{-1}$ or $\sim 17 \mathrm{meV}$ ). This feature, first observed by Ugawa et al. [92], has been interpreted as interband absorption in quasi-metallic nanotubes with curvature-induced gaps [92, 95-97] or absorption due to plasmon oscillations along the tube axis $[59,61,65,77,98-100]$, but a consensus has not been achieved [98].

\subsection{THz Emitters and Detectors}

There are several promising proposals of using carbon nanotubes for $\mathrm{THz}$ applications: a nanoklystron utilizing extremely efficient high-field electron emission from nanotubes $[23,48,101]$, devices based on negative differential conductivity in large-diameter semiconducting nanotubes [102, 103], high-frequency resonant-tunneling diodes [104] and Schottky diodes [105-108], as well as frequency multipliers [109,110], THz amplifiers [111], switches [112] and antennas [113]. THz detectors based upon antennas coupled to bundles [114-117] and individual [117,118] metallic SWCNTs have also been demonstrated.

$\ddagger$ Reprinted from Superlattices and Microstructures, 43, Portnoi M E, Kibis O V and Rosenau da Costa M, Terahertz applications of carbon nanotubes, 399-407, Copyright (2008), with permission 

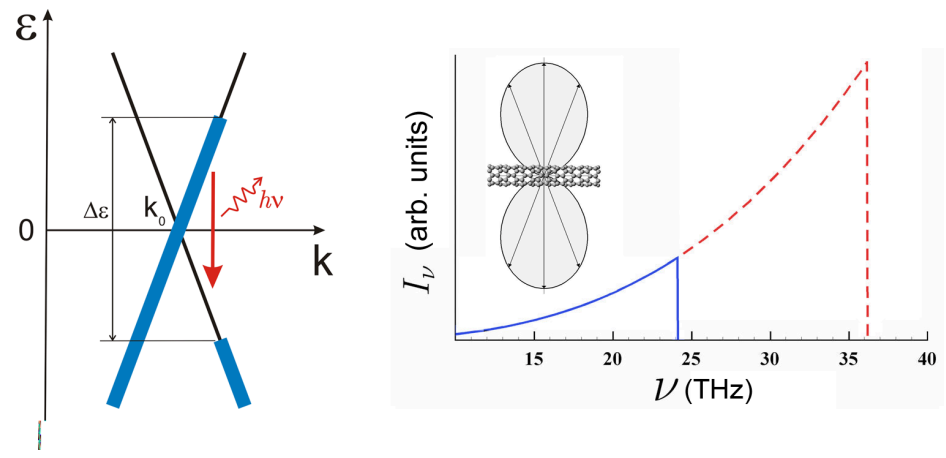

Figure 3. (a) The scheme of $\mathrm{THz}$ photon generation by hot carriers in quasi-metallic SWCNTs. (b) The calculated spectral density of spontaneous emission, $I_{\nu}$, as a function of frequency, $\nu$, for two values of applied voltage: solid line for $V=0.1 \mathrm{~V}$; dashed line for $V=0.15 \mathrm{~V}$.

A variety of proposals exist for using carbon nanotubes for $\mathrm{THz}$ emitters and detectors $[48,49,53-56,58,119]$. Kibis et al. [119] demonstrated theoretically that quasimetallic carbon nanotubes emit $\mathrm{THz}$ radiation when a potential difference is applied across their ends. The electric-field induced heating of the electron gas results in a population inversion of optically active states with an energy difference within the $\mathrm{THz}$ range (see figure 3). In the ballistic regime, the spontaneous emission spectra of all quasi-metallic have a universal dependence on the photon frequency [120], with the maximum of the spectral density of emission being controlled by the size of the applied voltage, which raises the possibility of utilizing this effect for high-frequency nanoelectronic devices. In figure 3 the spectral density is shown for two values of applied voltage.

It has been predicted that chiral carbon nanotubes can be used as the basis of tunable frequency multipliers $[49,53,54]$. An electric field applied normal to the nanotube axis gives rise to regions of the energy spectrum with negative effectivemass $[49,53,54]$, which are accessible in moderate electric fields. The effect of the negative effective mass also leads to an efficient frequency multiplication in the $\mathrm{THz}$ range, which can be controlled by the applied electric field.

Armchair SWCNTs are truly gapless, but by applying a magnetic field along the nanotube a bandgap can be opened [43,121-123]. For a $(10,10)$ SWCNT in a field of $10 \mathrm{~T}$ the bandgap corresponds to approximately $1.6 \mathrm{THz}$. It transpires that the same magnetic field which opens the bandgap also allows dipole optical transitions between the top valence subband and the lowest conduction subband $[49,55,56]$ which would otherwise be forbidden in the absence of a magnetic field $[124,125]$. In the presence of the field, the van Hove singularity in the reduced density of states leads to a very sharp absorption maximum near the band edge, which results in a very high sensitivity in the photocurrent to the photon frequency, whose peak frequency is tunable by the size of from Elsevier. 

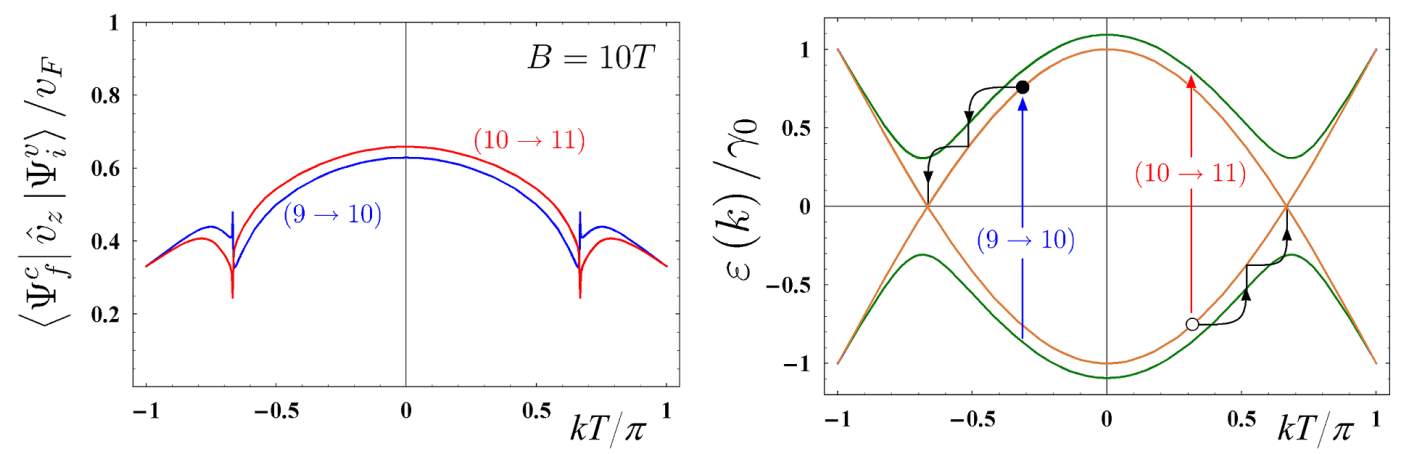

Figure 4. A scheme for creating a population inversion between the lowest conduction subband and the top valence subband of an armchair SWCNT in a magnetic field. The left plot shows the calculated matrix elements of the relevant dipole optical transitions polarized normally to the axis of a $(10,10)$ SWCNT. The right plot shows several energy subbands closest to the Fermi level and illustrates the creation of photoexcited carriers and their non-radiative thermalization. $\S$

the applied magnetic field. This scheme can also be used as a tunable emitter with a very narrow emission line. A population inversion can be achieved by optical pumping with the light polarized normally to the nanotube axis, as shown in figure 4.

In the absence of curvature, at $k=0$, optical transitions between the top valence subband and the bottom conduction subband in quasi-metallic SWCNTs are strictly forbidden by symmetry within the simple zone-folding model of the $\pi$-electron graphene spectrum. However, for zigzag quasi-metallic SWCNTs, dipole optical transitions are indeed allowed due to the gap opened in their energy spectrum by intrinsic curvature, which is of the order of $\mathrm{THz}[120,126]$. These transitions are several orders of magnitude larger than those previously considered in a model which neglects curvature[119]. As with the armchair nanotube, a magnetic field applied along the nanotube can be used to modify the optical selection rules. However in quasi metallic tubes, the magnetic field creates two different size bandgaps, and therefore two peaks in the absorption spectra. Arrays of armchair and quasi-metallic SWCNTs could be used as the building blocks of $\mathrm{THz}$ radiation detectors, which would have a high sensitivity in the photocurrent to photon frequency. Furthermore, since the bandgap of such SWCNTs can be controlled by the size of the applied magnetic field, such devices can be tunable.

Many-body (excitonic) effects, which dominate the optical properties of semiconducting SWCNTs [127-130], are also important in narrow-gap SWCNTs [131, 132]. However, due to the quasi-relativistic character of the free-particle dispersion near the band edge of the narrow-gap SWCNTs, there is a spectacular decrease in the exciton binding energy[131]. The binding energy scales with the bandgap and therefore, excitonic effects should not dominate in narrow-gap nanotubes. Hence, the proposed

$\S$ Reprinted from Superlattices and Microstructures, 43, Portnoi M E, Kibis O V and Rosenau da Costa M, Terahertz applications of carbon nanotubes, 399-407, Copyright (2008), with permission from Elsevier. 
$\mathrm{THz}$ applications of quasi-metallic nanotubes should be feasible.

Nemilentsau et al. [58] theoretically studied thermal radiation from an isolated finite-length carbon nanotube both in near- and far-fields. The formation of the discrete spectrum in metallic nanotubes in the $\mathrm{THz}$ range is demonstrated due to the reflection of strongly slowed-down surface-plasmon modes from nanotube ends.

It should be noted that there are some technological challenges that must be met before the aforementioned schemes can be experimentally realized. Proposals based on the superlattice properties of chiral nanotubes in a transverse electric field $[49,53,54]$ require using SWCNTs of a particular chirality, the growing of which remains a major technological challenge. However, post-growth techniques have recently progressed considerably. For instance, the ability to prepare macroscopic ensembles of SWCNTs enriched in a specific chirality [133-140] has already had significant impact in chiralitydependent spectroscopy studies $[100,126,141-145]$. With the rate of current progress, it is hoped that macroscopic devices of single-chirality-aligned nanotubes may be achievable within the next five years.

Detectors and emitters based upon magnetically controlled bandgaps are especially difficult to realize due to the high magnetic field strengths required. Furthermore, a key requirement for a detector is to have low contact resistance between the electrodes and the carbon nanotube as well as the ability to capture the measured $\mathrm{THz}$ radiation within a high-magnetic-field setup. For the case of a magnetically controlled $\mathrm{THz}$ emitter, suitable setups must be devised for the optical excitation of the carbon nanotubes as well the extraction of $\mathrm{THz}$ radiation from a suitably designed cavity in conjunction with a powerful magnet. Nevertheless transport experiments studying the influence of the magnetic field on the bandgap of quasi-metallic tubes have already been conducted [146]. Therefore, some progress in THz experiments can be envisaged for schemes based upon $\mathrm{THz}$ transitions across the magnetic-field-induced (or magnetic-field-controlled) gap in metallic (or quasi-metallic) carbon nanotubes.

\subsection{THz Antennae and Polarizers}

Nanotubes' desirable electronic properties and highly anisotropic optical properties make them ideally suited for antenna and polarizer applications in the $\mathrm{THz}$ range. Both single [113, 147-155] and multi-wall [156-158] carbon nanotube antennae operating in the $\mathrm{THz}$ regime have been studied extensively, and bundles/arrays of nanotubes [65, 159-163] have been shown to outperform tubes in isolation, demonstrating a far superior antenna efficiency [65]. The concept of a thermal nanoantenna was proposed by Nemilentsau et al. [58].

Polarization anisotropy in the $\mathrm{THz}$ range was first noted by Jeon and co-workers by using partially aligned nanotube films [59,60]. Akima et al. [61] also demonstrated anisotropy. More recently, using extremely well aligned ultralong carbon nanotubes, Ren and co-workers demonstrated that carbon nanotubes can be perfect $\mathrm{THz}$ polarizers [62]. Kyoung and co-workers used aligned multi-wall carbon nanotubes to demonstrate 

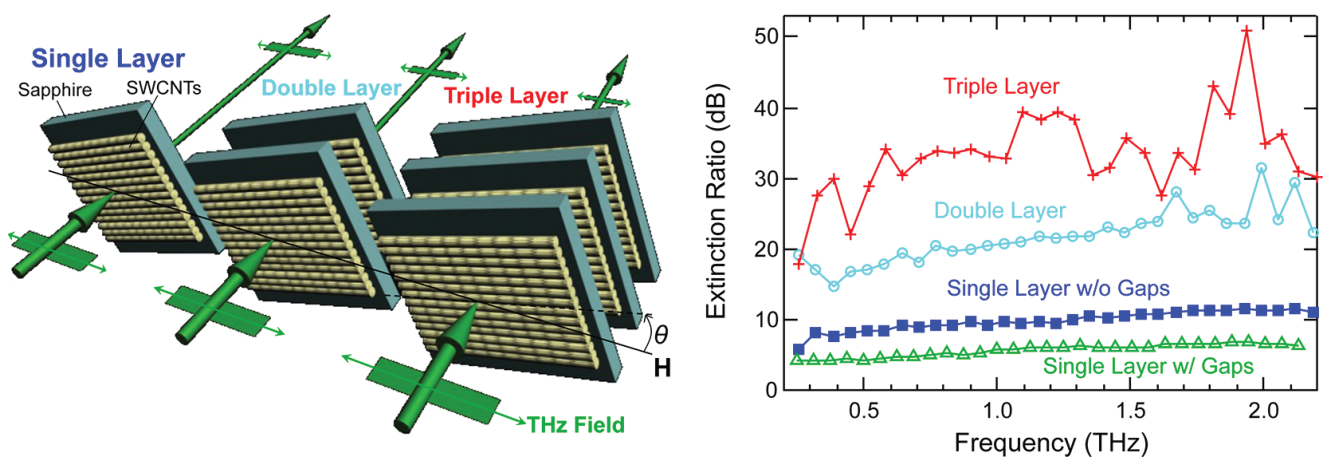

Figure 5. Scheme showing the use of multiple SWCNTs films to produce high performance polarizers. The extinction ratio of the $\mathrm{THz}$ polarizers with different thicknesses is shown as a function of frequency in the $\sim 0.4-2.2 \mathrm{THz}$ range. Reprinted (adapted) with permission from [64]. Copyright (2012) American Chemical Society.

similarly strong anisotropy [63].

More recently, Ren et al. showed that adding additional layers of films (as shown in figure 5) will enhance device performance considerably, achieving ideal broadband $\mathrm{THz}$ properties in a triple-stacked film: $99.9 \%$ degree of polarization and extinction ratios of $10^{-3}$ (or $30 \mathrm{~dB}$ ) from $\sim 0.4$ to $2.2 \mathrm{THz}$ [64], which is two orders of magnitude higher than that of a single layer. Not only do these multi-layer devices outperform wire-grid polarizers in the $\mathrm{THz}$ range, but since they are based on the intrinsic properties of the tubes (rather than geometric effects like wire-grid polarizers), such devices can operate beyond the $\mathrm{THz}$ range. Despite the macroscopic thickness of the layers, for polarizations normal to the nanotube axis there is almost zero absorption. However, in contrast to the Drude model, they show that when the polarization is parallel to the nanotube axis the transmittance decreases with increasing frequency. This is attributed to the existence of a peak in $\mathrm{THz}$ conductivity, which was discussed in section 2.1.

\subsection{THz Transistors}

The demonstration of ballistic transport [166] and linearity [167] in carbon nanotube field-effect transistors indicates that nanotubes are possible candidates to replace traditional silicon technology. By analyzing the influence of quantum capacitance, kinetic inductance, and ballistic transport on the high-frequency properties of nanotube transistors, Burke predicted a cutoff frequency of $80 \mathrm{GHz} / L$, where $L$ is the gate length in microns, opening up the possibility of a ballistic $\mathrm{THz}$ nanotube transistor [66]. Figure 6 shows how Burke's prediction compares against other technologies. Several other theoretical studies have also shown that nanotube field effect transistors can potentially operate in the $\mathrm{THz}$ frequency regime [90, 168-172].

Experimentally, several field-effect transistors based upon individual nanotubes,

|| Reprinted from Solid-State Electronics, 48, Burke P J, AC performance of nanoelectronics: towards a ballistic THz nanotube transistor, 1981, Copyright (2004), with permission from Elsevier. 


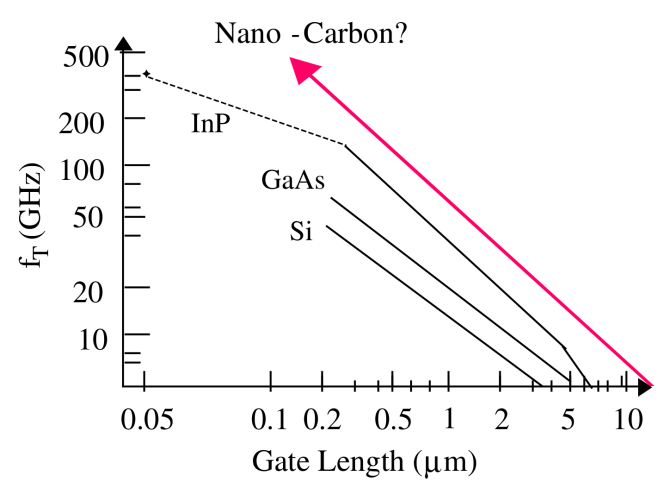

Figure 6. Transistor cutoff frequency, $f_{\mathrm{T}}$, versus gate length. References are: solid lines [164]; dashed line [165]; nano-carbon prediction [66]. ॥

operating in the low $\mathrm{GHz}$ range have been achieved [173-181]. However intrinsic cutoff frequencies in the $\mathrm{THz}$ regime have yet to be realized. The performance of such devices have been limited by parasitic effects and impedance mismatches. One approach to increase the extrinsic cutoff frequency is to utilize networks or arrays of aligned nanotubes [176,182-191]. Most recently, Che et al. [192] demonstrated that such an array of carbon nanotube transistors can achieve an extrinsic current-gain cutoff frequency of $25 \mathrm{GHz}$.

\section{THz Science and Technology of Graphene}

\subsection{Dynamic Conductivity}

The AC dynamics of Dirac fermions in graphene have attracted much theoretical attention - the influence of linear dispersions, two-dimensionality, and disorder has been extensively discussed by many theorists [38, 193-212]. However, the influence of electronelectron interactions on the optical conductivity of graphene is somewhat controversial. Theoretical studies using different methods have led to different conclusions as to the magnitude of many-body corrections to the Drude-like intraband optical conductivity (see, e.g., [201, 205, 206, 208] and references cited therein).

Experimentally, while a number of studies have confirmed the so-called universal optical conductivity $\sigma(\omega)=e^{2} / 4 \hbar$ for interband transitions in a wide spectra range [36, $37,213]$, successful experimental studies of the intraband conductivity have been reported only recently [214-221]. Also, two groups have used microscopy techniques to map out dynamic conductivity non-uniformity in the $\mathrm{GHz}$ [222] and $\mathrm{THz}$ [223] range and many successful dynamic conductivity measurements in magnetic fields (cyclotron resonance) exist [224-235]. 

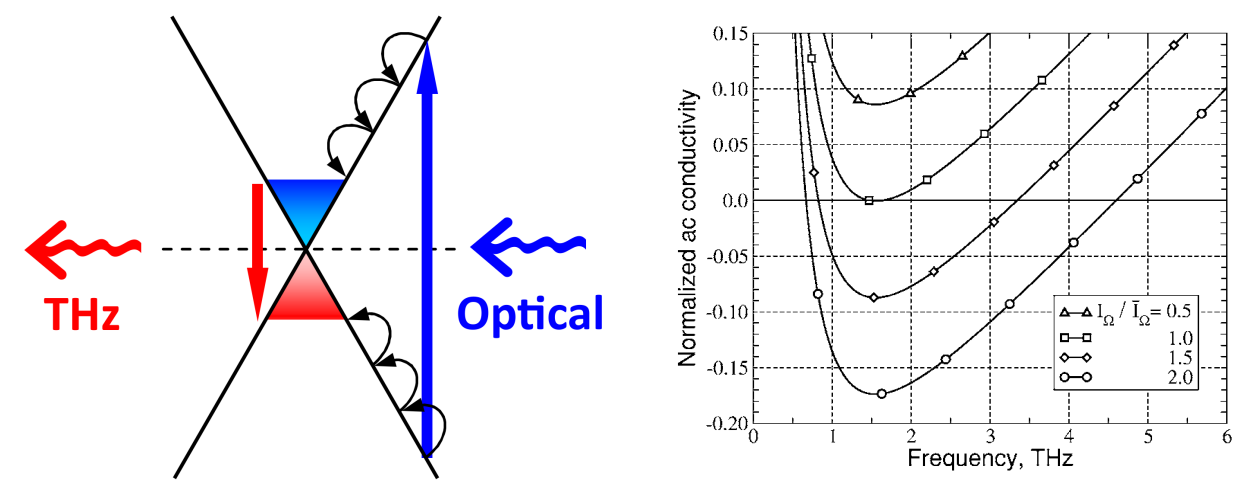

Figure 7. The left plot shows a scheme for creating a population inversion in graphene. Electrons and holes generated by optical pumping non-radiatively thermalize and aggregate towards the dirac points where they can recombine to emit $\mathrm{THz}$ radiation. The right plot shows the frequency dependence of normalized ac conductivity at different intensities of optical radiation, $I_{\Omega}$, with the photon energies $\hbar \Omega . \quad \bar{I}_{\Omega}$ is the threshold intensity of optical radiation. Reproduced with permission from [68]. Copyright [2007], AIP Publishing LLC.

\subsection{THz Gain and Lasing in Optically Excited Graphene}

An outstanding theoretical prediction is $\mathrm{THz}$ amplification in optically pumped graphene, proposed by Ryzhii and co-workers [68, 236-239]. They demonstrated that sufficiently strong optical pumping will result in population inversion, making the real part of the net AC conductivity negative, i.e., amplification (see figure 7). Due to the gapless energy spectrum, this negative $\mathrm{AC}$ conductivity takes place in the range of $\mathrm{THz}$ frequencies. They calculated the dynamic conductivity of a non-equilibrium 2D electron-hole system in graphene under interband optical excitation. Both interband and intraband transitions were taken into account in their model. It has been shown that under population inversion it is possible to achieve plasmon amplification through stimulated emission [240,241]. The predicted net plasmon gain for different electronhole densities at $300 \mathrm{~K}$ is shown in figure 8. Several plasmonic amplifiers and oscillations in graphene devices have been proposed for generating THz waves [241-245].

It has been predicted that $\mathrm{THz}$ lasing can be realized at room temperature in optically pumped lasers utilizing Fabry-Pérot resonators [237,246] and dielectric waveguides [247]. It has also been shown that population inversion can be achieved by current injection in graphene layer and multilayer graphene heterostuctures [248-251], and a novel voltage-tunable coherent $\mathrm{THz}$ emitters based upon multilayer grapheneboron-nitride heterostructures has been proposed by Mikhailov [252]. Freitag et al. observed thermal infrared emission from biased graphene [253], and Ramakrishnan et al. observed coherent $\mathrm{THz}$ emission from optically excited graphite [254].

A number of groups have performed ultrafast pump-probe spectroscopy to study carrier dynamics [215, 255-279], including optical-pump THz-probe experiments [215, 267-273, 278, 279], ultrafast $\mathrm{THz}$ photocurrent measurements [274], and THz-pump 

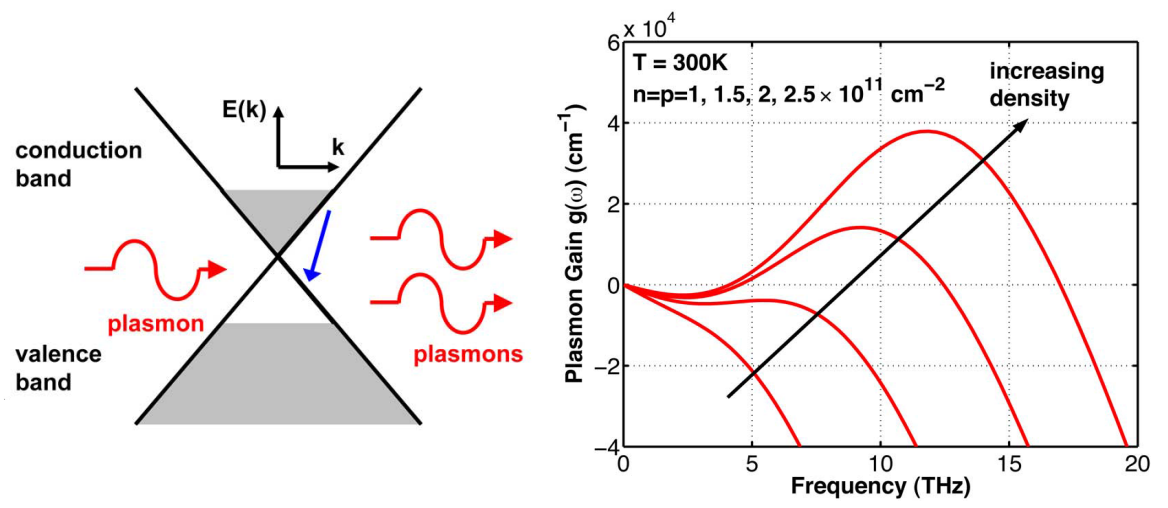

Figure 8. The left plot shows plasmon amplification through stimulated emission in population inverted graphene. The right plot shows the net plasmon gain (interband gain minus intraband loss) in graphene at $300 \mathrm{~K}$ plotted for different electron-hole densities $\left(n=p=1,1.5,2,2.5 \times 10^{11} \mathrm{~cm}^{-2}\right)$. The assumed values of the Fermi velocity and scattering time are $10^{8} \mathrm{~cm} / \mathrm{s}$ and 0.5 ps respectively. (c) [2008] IEEE. Reprinted, with permission, from [241].

and optical probe experiment [275]. Also, negative conductivity in the near-infrared region has been reported $[276,280]$. When describing carrier relaxation dynamics in graphene, both Coulomb-mediated carrier-carrier and electron-phonon scattering must be taken into account [239,281-291]. Electron-electron interactions such as Auger recombination and impact ionization, which are usually suppressed in conventional semiconductors, play an important role in the relaxation of photo-excited carriers in graphene. Theoretically, Auger recombination is prohibited in clean graphene [292] but is expected to become efficient in disordered graphene, which would lead to ultrafast recombination and prevent population inversion. On the other hand, impact ionization is predicted to lead to carrier multiplication [285], therefore enhancing the efficacy of graphene-based photodetectors. One critical question is whether optically created electrons and holes would thermalize before recombination and develop separate quasiFermi energies. Sun et al. [255, 265] and Tan et al. [275] argue that electron-electron interactions are so fast that the system attains a common Fermi energy very quickly. Breusing et al [258,264] and Li et al. [276] claim the opposite.

\subsection{THz Nonlinearities}

Mikhailov and Ziegler, using a semiclassical approximation, have shown that the dynamics of graphene in an AC electric field are intrinsically nonlinear, and efficient frequency multiplications for $\mathrm{THz}$ generation can be expected for microwave-driven graphene $[22,293,294]$. However, the current theories describing $\mathrm{THz}$ harmonic generation [22,293-296] do not take into account manybody Coulomb interactions and carrier-carrier scattering which are important when describing $\mathrm{THz}$ carrier dynamics in realistic graphene systems. Only very recently, Tani and co-workers performed THzpump optical-probe experiments to demonstrate ultrafast carrier-carrier scattering [275]. 


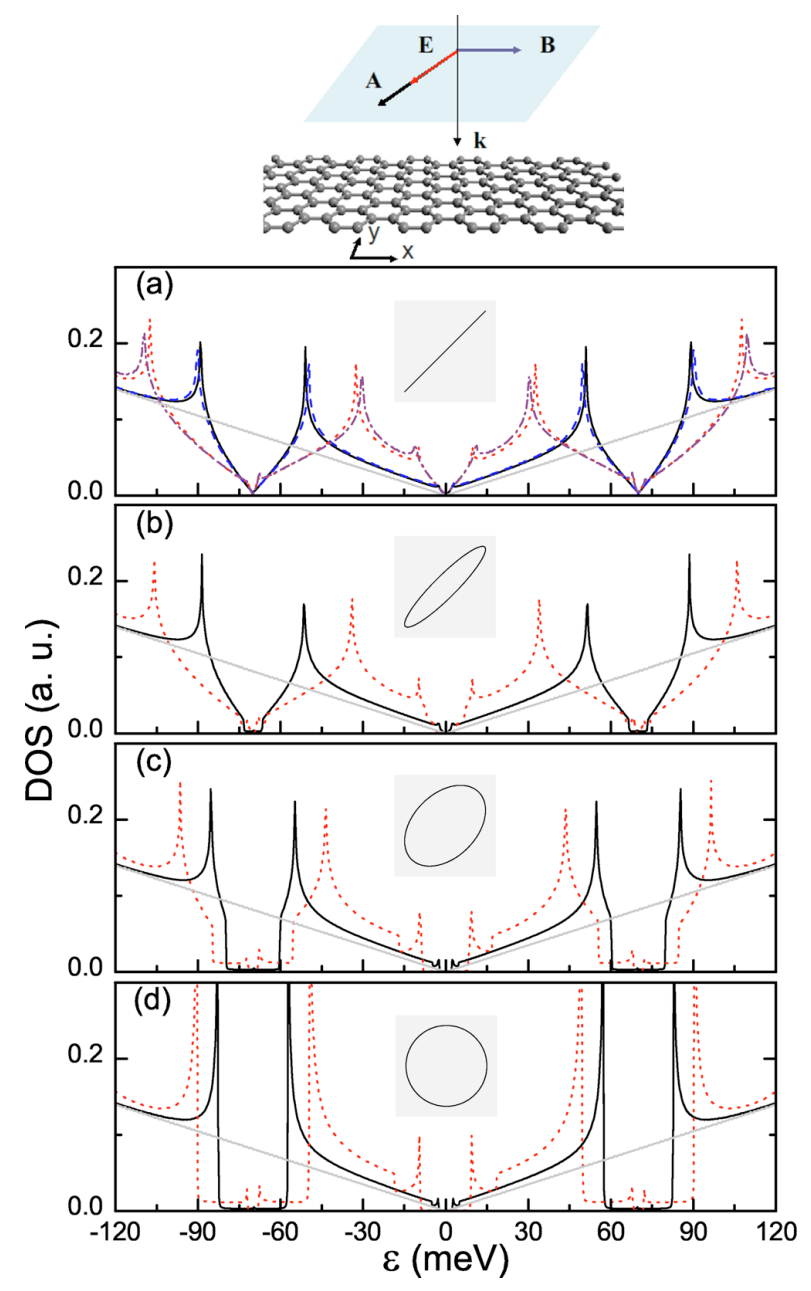

Figure 9. Scheme of the considered setup, where a laser field with $\hbar \Omega=140 \mathrm{meV}$ ( $\Omega$ is the laser frequency) and polarization $\varphi$ is applied perpendicular to a graphene monolayer. DOS for (a) linear, (b) $\varphi=0.125 \pi$, (c) $\varphi=0.375 \pi$, and (d) circular polarizations. The black solid line is for $I=3.2 \times 10^{6} \mathrm{~W} / \mathrm{cm}^{2}$ while the red dashed line corresponds to $I=1.3 \times 10^{7} \mathrm{~W} / \mathrm{cm}^{2}$. For linear polarization these results are compared with those of a tight-binding calculation for a system with $5 \times 10^{4}$ channels (blue dashed and purple dash-dotted lines). For reference the zero-field DOS is shown in solid gray. Reproduced with permission from [299]. Copyright [2011], AIP Publishing LLC.

The presence of such scattering is believed to explain the heavy suppression of nonlinear harmonic generation observed in recent experiments [297]. It has also recently been demonstrated that nonlinear optical effects in graphene can be greatly enhanced by its placement on a substrate possessing a negative dielectric constant [298].

Recent theoretical studies also suggest that graphene is an excellent material system for studying solid-state strong-field physics with various promising device possibilities. One of the predictions is that, when graphene is non-resonantly irradiated by a circularly-polarized laser field, an energy gap should appear at the Dirac point, whose magnitude is proportional to the laser intensity and inversely proportional to the frequency of the laser field [299-306]. This has tremendous device implications, since 
many optoelectronic applications require graphene to have a finite band gap, and this prediction provides a coherent and ultrafast means for tuning the bandgap. For example, Calvo and co-workers predicted that a long-wavelength (mid-infrared and $\mathrm{THz}$ ) laser field can produce significant band gaps in graphene [299] which can be modulated by the polarization of the field. Figure 9 shows how the dc density of states (DOS) varies with polarization. For a circularly-polarized mid-infrared laser field at a wavelength of $9 \mu \mathrm{m}$, the predicted size of the laser-induced gap is $23 \mathrm{meV}$ for an intensity of $3.2 \times 10^{6}$ $\mathrm{W} / \mathrm{cm}^{2}$ (which is easily achievable) and increases linearly with the intensity and the inverse of the laser frequency. Thus, the gaps are easily observable with reasonable intensities, and longer wavelengths (such as $\mathrm{THz}$ ) are preferable. It has also been shown that for circular polarizations, the same laser that opens the bandgap may also induce conducting chiral edge states in graphene [307]. It should also be noted that an additional degree of control can be achieved by lateral confinement. For a nanoribbon illuminated by linearly polarized light, it has been shown that a laser may or may not induce bandgaps. The opening of the gap is dictated by sample geometry, edge type and laser polarization angle relative to the sample edge [308].

Nonlinearities are also expected to appear in the dynamics of Dirac fermions in a magnetic field both in the semiclassical regime [309] and quantum regime [310]. Mikhailov predicts that due to the linear energy dispersion, the particle responds not only at the resonance frequency but generates a broad frequency spectrum around it. The linewidth of the cyclotron resonance is predicted to be very broad even in a perfectly pure material. A recent theoretical study by Yao and Belyanin [310] predicts that graphene possesses highest third-order nonlinear optical susceptibility $\chi^{(3)}$. These authors showed that Landau-quantized graphene (i.e., graphene in a perpendicular magnetic field) possesses an extremely high value of $\chi^{(3)}$, of the order of $10^{-2}$ esu in the mid/far-infrared range in a magnetic field of several tesla. This value is larger than reported $\chi^{(3)}$ values for any known materials by several orders of magnitude, and is a consequence of graphene's unique electronic properties and selection rules near the Dirac point.

\subsection{THz Plasmonics}

Graphene with its excellent electronic and optical properties makes it a promising candidate for the basis of future optoelectronic devices. Graphene based p-n junctions can be used to separate photo-excited charge carriers. However, the generation of photoexcited carriers is limited to the narrow region of the p-n junction and freestanding graphene only absorbs up to $2.3 \%$ of incident light $[36,37,311]$ these factors are hurdles to creating graphene-based devices with high efficiencies. However, absorbtion can be enhanced considerably using plasmonic nanostructures (up to 100\% [312,313]) to radiatively couple to surface plasmon modes, therefore improving the efficiency of graphene-based photodetectors. Furthermore, plasmonic nanostructures can be engineered to be resonant at particular wavelengths therefore opening the door to a 
new class of wavelength and polarization sensitive graphene-based devices.

Developing technologies to manipulate $\mathrm{THz}$ waves is an important goal in $\mathrm{THz}$ research. Several groups have already demonstrated that gated graphene acts as a modulator for $\mathrm{THz}$ waves [216,219-221,314]. Also, electro-optical modulations in graphene have been considered theoretically by Vasko et al. [212,315,316]. For both technological and scientific reasons, plasmons in graphene are attracting much attention. Their unique properties have been intensively studied both theoretically [317-335] and experimentally [70,336-340]. Many groups have started using gated graphene as well as graphene combined with metallic structures to manipulate $\mathrm{THz}$ waves [336, 341-344].

A surface plasmon is a coherent fluctuation of charge density in a conducting medium that is restricted to the interface formed between two materials, whose real part of their permittivities differ in sign. Optical excitation of this mode requires that one must provide both the correct energy and in-plane momentum. However, in general, the in-plane momentum of the surface plasmon exceeds that of the photon, and therefore, plane-wave radiation cannot couple to a flat surface. However, momentum matching can be achieved by several schemes, such as prism-coupling using attenuated total internal reflection [334,345] and by grating coupling [332,334,346-349], where the periodicity of the grating provides the additional in-plane momentum needed to excite the surface plasmon. An alternative approach is to spatially modulate graphene's conductivity. Graphene's charge carrier density and hence optical conductivity can be modified by external electric fields and strain, i.e., corrugation. Modulating the optical conductivity in a periodic manner allows the optical coupling to surface plasmons $[328,330-332,334]$. The periodicity of patterned graphene structures such as arrays of nanoribbons $[334,336,350]$ and graphene disks [312,342] can also be used.

Rather unusually, graphene can support both transverse magnetic and transverse electric [322] plasmon modes in the $\mathrm{THz}$ regime, the latter is absent in conventional 2D systems with parabolic electron dispersion. Graphene nanoribbons support both edge and waveguide $\mathrm{THz}$ surface plasmon modes, the number of which is a function of ribbon width and frequency [325]. Graphene plasmons can be tuned by electronic gating $[322,336,351,352]$ as well as by changing the surrounding dielectric environment. The plasmon lifetimes can be extended by increasing the doping level [324], and plasmons can be guided by $\mathrm{p}-\mathrm{n}$ junctions [351]. These properties have lead to several novel device proposals $[69,71,326,336,346,353-355]$, ranging from waveguides [356], switches $[343,345,357,358]$, and other control devices [221, 344, 359, 360] through to sensors [358], tunable filters [361-363] and generators of $\mathrm{THz}$ radiation [241-245].

The first proposals using graphene for antenna applications use graphene as a control element [364]. It was shown that by modulating graphene's conductivity via electronic gating allows the radiation pattern of the deposited metallic dipole antennas to be controlled. A beam reconfigurable antenna was also designed [365], using switchable high impedance surfaces. Several tunable graphene-based antenna schemes using plasmon modes have been developed [327,366-369]. Such plasmonic devices offer the possibility of tunable antenna which can be miniaturized down to the 


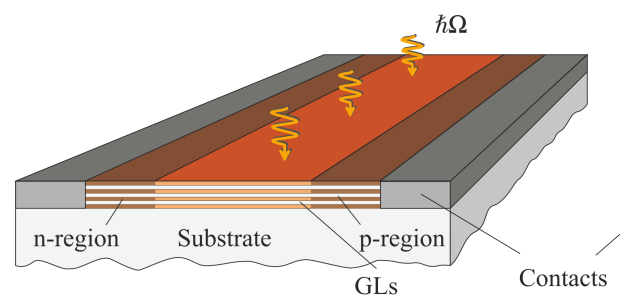

(a)

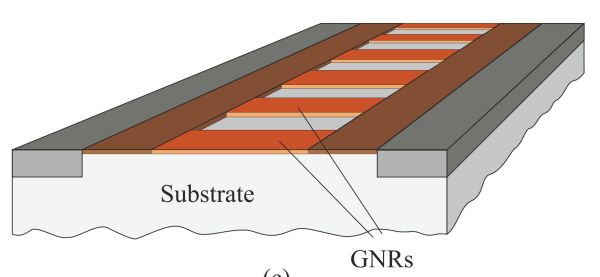

(c)

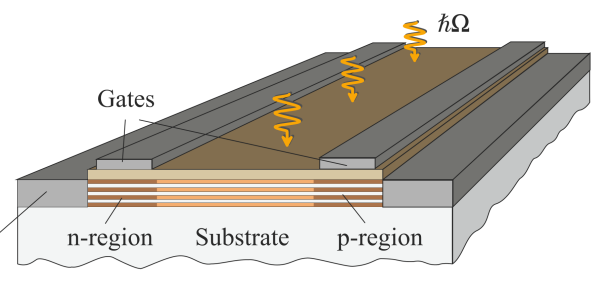

(b)

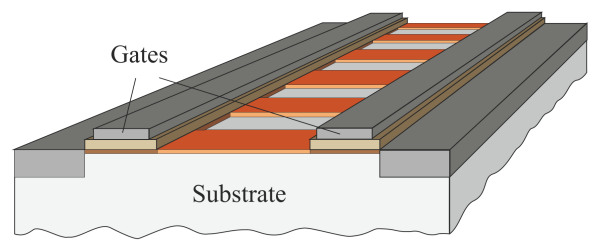

(d)

Figure 10. Schematic views of multi-layer graphene p-i-n photodiodes with (a) chemically doped $\mathrm{p}$ - and n-regions and (b) electrically induced $\mathrm{p}$ - and n-regions (electrical doping), as well as of graphene nanoribbons p-i-n photodiodes with (c) chemically doped p- and n-regions and (d) electrically induced p-and n-regions, respectively. From [371] with kind permission from Springer Science+Business Media B.V.

nanometer scale, and the recent development of Leaky-Wave Antenna has shown that fixed-frequency beamscanning is also realizable [370].

\subsection{Detectors}

Several schemes using graphene-based heterostructures as $\mathrm{THz}$ detectors have been proposed, ranging from devices utilizing randomly stacked multi-layer graphene [371374], bilayer graphene [375], as well as devices based upon arrays of graphene nanoribbons [371,376-378] and graphene-based heterostructures which make use of plasmonic effects [378,379]. Some of the proposed devices are shown in figure 10.

Experimentally, several graphene single- and few-layer photodetectors have been developed, which can operate at room temperature [380-383]. In such devices, the generation of photocurrent is attributed to charge separation due to internal fields, which arise at the graphene-metal interfaces [384-386] and the photo-thermoelectric effect. Recently, broadband $(0.76$ to $33 \mathrm{THz}$ ) graphene transistor detectors have been achieved [67], which can be tuned by the strength of the applied magnetic field. Plasmonic nanostructures have also been shown to enhance the efficacy of photodetectors and allow such devices to be wavelength and polarization sensitive [346].

Hot electrons have also been utilized in bolometric $\mathrm{THz}$ devices operating at cryogenic temperatures made from graphene [387-389] and bi-layer graphene [390, 391]. Bi-layer graphene is a gapless semiconductor with parabolic dispersion [392], but a band gap can be opened between the conduction and valence bands by applying a potential across the two layers. The band gap and the temperature-dependence 
of the resistivity is tunable by the strength of the applied field. These properties have been exploited to create bolometric devices that operate at $\mathrm{THz}$ frequencies [390,391]. Other bolometric devices use Johnson-noise thermometry [387-389], which have sufficient energy resolutions to detect individual $\mathrm{THz}$ photons. Room temperature bolometers based on graphene-graphene nanoribbon hybrid heterostructures have also been theoretically proposed [393].

The optically induced breakdown of quantum Hall effect has been utilized to create detectors which operate at liquid nitrogen temperatures [394], and reduced graphene oxide and graphene nanoribbons infrared photodetectors have also been demonstrated [395]. Karch and co-workers reported the observation of the Dynamic Hall Effect and Chiral Edge Currents in THz driven graphene [396, 397] as well as the magnetic quantum ratchet effect [398], and room temperature $\mathrm{THz}$ detectors have also been achieved using antenna-coupled graphene based devices [382,399].

\section{Future Perspectives}

Nanocarbon $\mathrm{THz}$ technology has great potential for development in the coming years. Graphene and carbon nanotubes could serve as the basis for new and efficient $\mathrm{THz}$ sources and detectors, which are tunable, compact and operate at room temperature. Their desirable electronic and optical properties make them ideally suited for $\mathrm{THz}$ antenna and polarizer applications, as well holding the promise of ballistic $\mathrm{THz}$ transistors that could supersede traditional silicon technology.

Though significant progress has been made in the field of terahertz science and technology of carbon nanomaterials there are still many challenges which lie ahead. One of the major limiting factors to the commercialization of graphene based devices is the viability of its production. Though the synthesis of graphene by both chemical vapour deposition and from $\mathrm{SiC}$ offers much promise current cost, sample quality, uniformity, prevalence of defects and unwanted dopants are all issues that need improvement. Furthermore, it would be desirable to have full control over the number of layers, and their crystallographic orientation. Similarly the production methods of carbon nanotubes must also be improved, as many carbon nanotube based $\mathrm{THz}$ devices require highly aligned arrays of nanotubes of a certain chirality which remains a major technological challenge. The performance of graphene and carbon nanotube transistors devices are limited by parasitic effects and impedance mismatches. For THz operation to be realized efforts must be made to reduce source, drain, and gate resistances as well as searching for alternative substrates. An other major obstacle to be overcome for graphene photodetectors is their low responsivity which arises from graphene's low absorption and small area for the generation of photoexcited carriers. However, plasmonic nanostructures and waveguide structures offers the promise of enhanced sensitivity.

From the theoretical conception of the quantum cascade laser [400] through to its first experimental manifestation [401] took over two decades and significant technological 
advances to bring about its fruition. Similarly there is much hope that the inevitable progress in technology will likewise lead to further experimental breakthroughs in the field of terahertz science and technology of carbon nanomaterials in the near future.

\section{Acknowledgments}

R.R.H acknowledges financial support from URCO (through Grant No. 17 N 1TAY121TAY13 and $15 \mathrm{~F} \mathrm{U} / \mathrm{S}$ 1TAY13-1TAY14). J.K. acknowledges support from the National Science Foundation (through Grants No. OISE-0530220 and EEC-0540832), Department of Energy BES Program (through Grant No. DE-FG02-06ER46308), and the Robert A. Welch Foundation (through Grant No. C-1509). M.E.P was supported by the EU FP7 ITN NOTEDEV (through Grant No. FP7-607521) and FP7 IRSES projects QOCaN (through Grant No. FP7-316432), CANTOR (through Grant No. FP7-612285), and InterNoM (through Grant No. FP7-612624).

\section{References}

[1] Kroto H W, Heath J R, O'Brien S C, Curl R F and Smalley R E 1985 Nature 318 162-163

[2] Greiner N R, Phillips D S, Johnson J D and Volk F 1988 Nature 333 440-2

[3] Mochalin V N, Shenderova O, Ho D and Gogotsi Y 2012 Nature Nanotechnol. 7 11-23

[4] Iijima S 1991 Nature 354 56-8

[5] Novoselov K S, Geim A K, Morozov S, Jiang D, Zhang Y, Dubonos S, Grigorieva I and Firsov A 2004 Science 306 666-669

[6] Geim A K and Novoselov K S 2007 Nature Materials 6 183-91

[7] Lee M and Wanke M C 2007 Science 316 64-65

[8] Globus T R, Woolard D L, Khromova T, Crowe T W, Bykhovskaia M, Gelmont B, Hesler J and Samuels A C 2003 J. Bio. Phys. 29 89-100

[9] Parthasarathy R, Bykhovski A, Gelmont B, Globus T, Swami N and Woolard D 2007 Phys. Rev. Lett. 98153906

[10] Powell J, Edwards G, Genzel L, Kremer F, Wittlin A, Kubasek W and Peticolas W 1987 Phys. Rev. A 353929

[11] Woolard D L, Globus T R, Gelmont B L, Bykhovskaia M, Samuels A C, Cookmeyer D, Hesler J L, Crowe T W, Jensen J O, Jensen J L et al. 2002 Phys. Rev. E 65051903

[12] Woolard D L, Loerop W R and Shur M S 2003 Terahertz Sensing Technology: Emerging scientific applications \& novel device concepts. Volume 2 vol 2 (World Scientific)

[13] Taday P F, Bradley I V, Arnone D D and Pepper M 2003 J. Pharm. Sci. 92 831-838

[14] Nahata A, Weling A S and Heinz T F 1996 Appl. Phys. Lett. 69 2321-2323

[15] Xu B, Hu Q and Melloch M R 1997 Appl. Phys. Lett. 71 440-442

[16] Ramian G 1992 Nucl. Instrum. Methods Phys. Res. Sect. A 318225

[17] Carr G L, Martin M C, McKinney W R, Jordan K, Neil G R and Williams G P 2002 Nature 420 153-156

[18] Köhler R, Tredicucci A, Beltram F, Beere H E, Linfield E H, Davies A G, Ritchie D A, Iotti R C and Rossi F 2002 Nature 417 156-159

[19] Ferguson B and Zhang X C 2002 Nature Mater. 1 26-33

[20] Avrutin E A and Portnoi M E 1988 Sov. Phys. Semicond 22 968-969

[21] Kruglyak V V and Portnoi M E 2005 Techn. Phys. Lett. 31 1047-1048

[22] Mikhailov S A 2007 Europhys. Lett. 7927002

[23] Dragoman D and Dragoman M 2004 Prog. Quantum Electron. 28 1-66 
[24] Tonouchi M 2007 Nature Photon. 1 97-105

[25] Shumyatsky P and Alfano R R 2011 J. Biomed. Opt. 16033001

[26] Ulbricht R, Hendry E, Shan J, Heinz T F and Bonn M 2011 Rev. Mod. Phys. 83 543-586

[27] Nagatsuma T 2011 IEICE Electron. Express 8 1127-1142

[28] Saito R, Dresselhaus G and Dresselhaus M S 1998 Physical Properties of Carbon Nanotubes (London: Imperial College Press)

[29] Wallace P 1947 Phys. Rev. 71622

[30] Novoselov K, Geim A K, Morozov S, Jiang D, Grigorieva M K I, Dubonos S and Firsov A 2005 nature 438 197-200

[31] Neto A H C, Guinea F, Peres N M R, Novoselov K S and Geim A K 2009 Rev. Mod. Phys. 81 109

[32] Katsnelson M, Novoselov K and Geim A 2006 Nature Phys. 2 620-625

[33] Katsnelson M I and Novoselov K 2007 Solid State Commun. 143 3-13

[34] Ando T, Nakanishi T and Saito R 1998 J. Phys. Soc. Jpn. 67 2857-2862

[35] Zhang Y, Tan Y W, Stormer H L and Kim P 2005 Nature 438 201-204

[36] Mak K F, Sfeir M Y, Wu Y, Lui C H, Misewich J A and Heinz T F 2008 Phys. Rev. Lett. 101 196405

[37] Nair R R, Blake P, Grigorenko A N, Novoselov K S, Booth T J, Stauber T, Peres N M R and Geim A K 2008 Science 3201308

[38] Ando T, Zheng Y and Suzuura H 2002 J. Phys. Soc. Jpn. 71 1318-1324

[39] Nakada K, Fujita M, Dresselhaus G and Dresselhaus M S 1996 Phys. Rev. B 5417954

[40] Zheng H, Wang Z, Luo T, Shi Q and Chen J 2007 Phys. Rev. B 75165414

[41] Brey L and Fertig H 2006 Phys. Rev. B 73235411

[42] Dresselhaus M S, Dresselhaus G and Avouris P (eds) 2001 Carbon Nanotubes: Synthesis, Structure, Properties, and Applications (Topics in Applied Physics no 18) (Berlin: Springer)

[43] Reich S, Thomsen C and Maultzsch J 2004 Carbon Nanotubes: Basic Concepts and Physical Properties (Weinheim: Wiley-VCH)

[44] Anantram M P and Léonard F 2006 Rep. Prog. Phys. 69507

[45] Hamada N, Sawada S and Oshiyama A 1992 Phys. Rev. Lett. 68 1579-1581

[46] Kane C L and Mele E J 1997 Phys. Rev. Lett. 78 1932-1935

[47] Gunlycke D, Lambert C, Pettifor D, Briggs G and Jefferson J 2006 Europhys. Lett. 73759

[48] Portnoi M E, Kibis O V and Rosenau da Costa M 2006 Proc. SPIE 6328632805

[49] Portnoi M E, Kibis O V and Rosenau da Costa M 2008 Superlattices and Microstructures 43 399-407

[50] Mikhailov S A 2009 Microelectron. J. 40 712-715

[51] Ren L, Zhang Q, Nanot S, Kawayama I, Tonouchi M and Kono J 2012 J. Infrared Milli. Terahertz Waves 33 846-860

[52] Otsuji T, Boubanga-Tombet S A, Satou A, Fukidome H, Suemitsu M, Sano E, Popov V, Ryzhii M and Ryzhii V 2012 J. Phys. D: Appl. Phys. 45303001

[53] Kibis O, Parfitt D and Portnoi M 2005 Phys. Rev. B 71035411

[54] Kibis O, Malevannyy S, Huggett L, Parfitt D and Portnoi M 2005 Electromagnetics 25 425-435

[55] Portnoi M E, Rosenau da Costa M, Kibis O V and Shelykh I A 2009 Int. J. Mod. Phys. B 23 $2846-2850$

[56] Batrakov K G, Kibis O V, Kuzhir P P, Da Costa M R and Portnoi M E 2010 Journal of Nanophotonics 4 041665-041665

[57] Batrakov K G, Kibis O V, Kuzhir P P, Maksimenko S A, Rosenau da Costa M and Portnoi M E 2010 Physica B 405 3054-3056

[58] Nemilentsau A M, Slepyan G Y and Maksimenko S A 2007 Phys. Rev. Lett. 99147403

[59] Jeon T I, Kim K J, Kang C, Oh S J, Son J H, An K H, Bae D J and Lee Y H 2002 Appl. Phys. Lett. $803403-3405$

[60] Jeon T I, Kim K J, Kang C, Maeng I H, Son J H, An K H, Lee J Y and Lee Y H 2004 J. Appl. 
Phys. 95 5736-5740

[61] Akima N, Iwasa Y, Brown S, Barbour A M, Cao J, Musfeldt J L, Matsui H, Toyota N, Shiraishi M, Shimoda H and Zhou O 2006 Adv. Mater. 18(9) 1166-1169

[62] Ren L, Pint C L, Booshehri L G, Rice W D, Wang X, Hilton D J, Takeya K, Kawayama I, Tonouchi M, Hauge R H and Kono J 2009 Nano Lett. 9 2610-2613

[63] Kyoung J, Jang E Y, Lima M D, Park H R, Robls R O, Lepro X, Kim Y H, Baughman R H and Kim D S 2011 Nano Lett. 11 4227-4231

[64] Ren L, Pint C L, Arikawa T, Takeya K, Kawayama I, Tonouchi M, Hauge R H and Kono J 2012 Nano Lett. 12 787-790

[65] Ren L, Zhang Q, Pint C, Wójcik A, Bunney Jr M, Arikawa T, Kawayama I, Tonouchi M, Hauge R, Belyanin A et al. 2013 Phys. Rev. B 87161401

[66] Burke P J 2004 Solid-State Electronics 48 1981-1986

[67] Kawano Y 2013 Nanotechnology 24214004

[68] Ryzhii V, Ryzhii M and Otsuji T 2007 J. Appl. Phys. $101083114-083114$

[69] Grigorenko A N, Polini M and Novoselov K S 2012 Nature Photon. 6 749-758

[70] Gao W, Shi G, Jin Z, Shu J, Zhang Q, Vajtai R, Ajayan P M, Kono J and Xu Q 2013 Nano Lett. 13 3698-3702

[71] Low T and Avouris P 2014 ACS Nano 8 1086-1101

[72] Sablikov V A and Shchamkhalova B S 1997 JETP Lett. 66 41-46

[73] Rosch A and Andrei N 2000 Phys. Rev. Lett. 85 1092-1095

[74] Ando T 2002 J. Phys. Soc. Jpn. 71 2505-2511

[75] Burke P J 2002 IEEE Trans. Nanotechnol. 1 129-144

[76] Pustilnik M, Khodas M, Kamenev A and Glazman L I 2006 Phys. Rev. Lett. 96(19) 196405

[77] Nakanishi T and Ando T 2009 J. Phys. Soc. Jpn. 78114708

[78] Giamarchi T 2004 Quantum Physics in One Dimension (Oxford: Oxford University Press)

[79] Tomonaga S i 1950 Progr. Theor. Phys. 5 544-569

[80] Luttinger J M 1963 J. Math. Phys. 4 1154-1162

[81] Egger R and Gogolin A O 1997 Phys. Rev. Lett. 79 5082-5085

[82] Kane C, Balents L and Fisher M P A 1997 Phys. Rev. Lett. 795086

[83] Bockrath M, Cobden D H, Lu J, Rinzler A G, Smalley R E, Balents L and McEuen P L 1999 Nature $397598-601$

[84] Yao Z, Postma H W C, Balents L and Dekker C 1999 Nature 402 273-276

[85] Postma H W C, de Jonge M, Yao Z and Dekker C 2000 Phys. Rev. B 62 R10653

[86] Lee J, Eggert S, Kim H, Kahng S J, Shinohara H and Kuk Y 2004 Phys. Rev. Lett. 93166403

[87] Tarkiainen R, Ahlskog M, Penttilä J, Roschier L, Hakonen P, Paalanen M and Sonin E 2001 Phys. Rev. B 64195412

[88] Bachtold A, De Jonge M, Grove-Rasmussen K, McEuen P, Buitelaar M and Schönenberger C 2001 Phys. Rev. Lett. 87166801

[89] Zhong Z, Gabor N M, Sharping J E, Gaeta A L and McEuen P L 2008 Nat. Nanotechnol. 3 201-205

[90] Kienle D and Léonard F 2009 Phys. Rev. Lett. 103026601

[91] Bommeli F, Degiorgi L, Wachter P, Bacsa W S, de Heer W A and Forro L 1996 Solid State Commun. 99 513-517

[92] Ugawa A, Rinzler A G and Tanner D B 1999 Phys. Rev. B 60 R11305-R11308

[93] Itkis M E, Niyogi S, Meng M E, Hamon M A, Hu H and Haddon R C 2002 Nano Lett. 2 155-159

[94] Jeon T I, Son J H, An K H, Lee Y H and Lee Y S 2005 J. Appl. Phys. 98034316 (pages 4)

[95] Borondics F, Kamarás K, Nikolou M, Tanner D B, Chen Z H and Rinzler A G 2006 Phys. Rev. B 74045431

[96] Nishimura H, Minami N and Shimano R 2007 Appl. Phys. Lett. 91011108

[97] Kampfrath T, von Volkmann K, Aguirre C M, Desjardins P, Martel R, Krenz M, Frischkorn C, Wolf M and Perfetti L 2008 Phys. Rev. Lett. 101267403 
[98] Slepyan G Y, Shuba M V, Maksimenko S A, Thomsen C and Lakhtakia A 2010 Phys. Rev. B 81 205423

[99] Shuba M, Paddubskaya A, Plyushch A, Kuzhir P, Slepyan G Y, Maksimenko S, Ksenevich V, Buka P, Seliuta D, Kasalynas I et al. 2012 Phys. Rev. B 85165435

[100] Zhang Q, Hároz E H, Jin Z, Ren L, Wang X, Arvidson R S, Lüttge A and Kono J 2013 Nano Lett. 13 5991-5996

[101] Manohara H M, Bronikowski M J, Hoenk M, Hunt B D and Siegel P H 2005 J. Vac. Sci. Technol. B 23 157-161

[102] Maksimenko A S and Slepyan G Y 2000 Phys. Rev. Lett. 84362

[103] Pennington G and Goldsman N 2003 Phys. Rev. B 68045426

[104] Dragoman D and Dragoman M 2004 Physica E 24 282-289

[105] Odintsov A A 2000 Phys. Rev. Lett. 85 150-3

[106] Léonard F and Tersoff J 2000 Phys. Rev. Lett. 854767

[107] Yang M, Teo K, Milne W and Hasko D 2005 Appl. Phys. Lett. 87 253116-253116

[108] Lu C, An L, Fu Q, Liu J, Zhang H and Murduck J 2006 Appl. Phys. Lett. 88 133501-133501

[109] Slepyan G Y, Maksimenko S, Kalosha V, Herrmann J, Campbell E and Hertel I 1999 Phys. Rev. A $60 \mathrm{R} 777$

[110] Slepyan G Y, Maksimenko S, Kalosha V, Gusakov A and Herrmann J 2001 Phys. Rev. A 63 053808

[111] Dragoman D and Dragoman M 2005 Physica E 25 492-496

[112] Dragoman M, Cismaru A, Hartnagel H and Plana R 2006 Appl. Phys. Lett. 88 073503-073503

[113] Slepyan G Y, Shuba M V, Maksimenko S A and Lakhtakia A 2006 Phys. Rev. B 73195416

[114] Polizzi E, Yngvesson K, Nicholson J, Adams S, Chan C, Fu K and Zannoni R 2008 Appl. Phys. Lett. 92 033105-033105

[115] Yngvesson K, Fu K, Fu B, Zannoni R, Nicholson J, Adams S, Ouarraoui A, Donovan J and Polizzi E 2008 Experimental detection of terahertz radiation in bundles of single wall carbon nanotubes Proc. 19th Int. Symp. Space THz Techn pp 304-313

[116] Carrion E, Muthee M, Donovan J, Zannoni R, Nicholson J, Polizzi E and Yngvesson K S 2009 New results on terahertz detection by carbon nanotubes Proc. 20th Int. Symp. Space THz Technology, Charlottesville

[117] Carrion E, Muthee M, Chen Z, Nicholson J, Polizzi E and Yngvesson S 2010 Single wall carbon nanotube (swcnt) devices as thz detectors and mixers Twenty-First International Symposium on Space Terahertz Technology vol 1 pp 312-317

[118] Chudow J D, Santavicca D F, McKitterick C B, Prober D E and Kim P 2012 Appl. Phys. Lett. 100163503

[119] Kibis O V, Rosenau da Costa M and Portnoi M E 2007 Nano Lett. 7 3414-3417

[120] Hartmann R R and Portnoi M E 2011 Optoelectronic Properties of Carbon-based Nanostructures: Steering electrons in graphene by electromagnetic fields (Saarbrücken: LAP Lambert Academic Publishing) ISBN 3844328580

[121] Ajiki H and Ando T 1993 J. Phys. Soc. Jpn. 62 1255-1266

[122] Kono J and Roche S 2006 Magnetic properties Carbon Nanotubes: Properties and Applications ed O'Connell M J (Boca Raton: CRC Press, Taylor \& Francis Group) chap 5, pp 119-151

[123] Kono J, Nicholas R J and Roche S 2008 High magnetic field phenomena in carbon nanotubes Carbon Nanotubes: Advanced Topics in the Synthesis, Structure, Properties and Applications ed Jorio A, Dresselhaus G and Dresselhaus M S (Berlin: Springer) pp 393-421 ISBN 978-3540-72864-1

[124] Milošević I, Vukovic T, Dmitrovic S and Damnjanović M 2003 Phys. Rev. B 67165418

[125] Jiang J, Saito R, Grüneis, Dresselhaus G and Dresselhaus M S 2004 Carbon 42 3169-3176

[126] Hároz E H, Duque J G, Tu X, Zheng M, Walker A R H, Hauge R H, Doorn S K and Kono J 2013 Nanoscale 5 1411-1439

[127] Ando T 1997 J. Phys. Soc. Jpn. 66 1066-1073 
[128] Wang F, Dukovic G, Brus L E and Heinz T F 2005 Science 308838

[129] Maultzsch J, Pomraenke R, Reich S, Chang E, Prezzi D, Ruini A, Molinari E, Strano M S, Thomsen C and Lienau C 2005 Phys. Rev. B 72241402

[130] Shaver J and Kono J 2007 Laser 83 Photonics Rev. 1 260-274

[131] Hartmann R R, Shelykh I A and Portnoi M E 2011 Phys. Rev. B 84035437

[132] Portnoi M, Downing C, Hartmann R and Shelykh I 2013 Excitons and interband terahertz transitions in narrow-gap carbon nanotubes Electromagnetics in Advanced Applications (ICEAA), 2013 International Conference on pp 231-234

[133] Zheng M, Jagota A, Strano M S, Santos A P, Barone P, Chou S G, Diner B A, Dresselhaus M S, Mclean R S, Onoa G B et al. 2003 Science 302 1545-1548

[134] Zheng M, Jagota A, Semke E D, Diner B A, McLean R S, Lustig S R, Richardson R E and Tassi N G 2003 Nat. Mat. 2 338-342

[135] Arnold M S, Stupp S I and Hersam M C 2005 Nano Lett. 5 713-718

[136] Arnold M S, Green A A, Hulvat J F, Stupp S I and Hersam M C 2006 Nature Nanotechnol. 1 $60-65$

[137] Ghosh S, Bachilo S M and Weisman R B 2010 Nature Nanotechnol. 5 443-450

[138] H Liu D Nishide T T and Kataura H 2011 Nat. Commun. 2309

[139] Tu X, Hight Walker A R, Khripin C Y and Zheng M

[140] Khripin C Y, Fagan J A and Zheng M 2013 J. Am. Chem. Soc. 135 6822-6825

[141] Hároz E H, Rice W D, Lu B Y, Ghosh S, Hauge R H, Weisman R B, Doorn S K and Kono J 2010 ACS Nano 4 1955-1962

[142] Duque J G, Chen H, Swan A K, Shreve A P, Kilina S, Tretiak S, Tu X, Zheng M and Doorn S K 2011 ACS Nano 5 5233-5241

[143] Hároz E H, Duque J G, Rice W D, Densmore C G, Kono J and Doorn S K 2011 Phys. Rev. B 84(12) 121403

[144] Hároz E H, Duque J G, Lu B Y, Nikolaev P, Arepalli S, Hauge R H, Doorn S K and Kono J 2012 J. Am. Chem. Soc. 134 4461-4464

[145] Lim Y S, Nugraha A R T, Cho S J, Noh M Y, Yoon E J, Liu H, Kim J H, Telg H, Hroz E H, Sanders G D, Baik S H, Kataura H, Doorn S K, Stanton C J, Saito R, Kono J and Joo T 2014 Nano Lett. 14 1426-1432

[146] Fedorov G, Tselev A, Jiménez D, Latil S, Kalugin N G, Barbara P, Smirnov D and Roche S 2007 Nano Lett. 7 960-964

[147] Hanson G W 2005 IEEE Trans. Antennas. Propag. 53 3426-3435

[148] Hanson G W 2006 IEEE Trans. Antennas. Propag. 54 76-81

[149] Burke P J, Li S and Yu Z 2006 IEEE Trans. Nanotechnol. 5 314-334

[150] Slepyan G Y, Shuba M, Nemilentsau A and Maksimenko S 2008 Electromagnetic theory of nanodimensional antennas for terahertz, infrared and optical regimes Mathematical Methods in Electromagnetic Theory, 2008. MMET 2008. 12th International Conference on (IEEE) pp $118-123$

[151] Hao J and Hanson G W 2006 IEEE Trans. Nanotechnol. 5 766-775

[152] Wang Y and Wu Q 2008 Chinese Optics Letters 6 770-772

[153] Yue W, Qun W, Xun-Jun H, Shao-Qing Z and Lei-Lei Z 2009 Chinese Physics B 181801

[154] Fichtner N, Zhou X and Russer P 2008 Advances in Radio Science 6 209-211

[155] Jornet J M and Akyildiz I F 2010 Graphene-based nano-antennas for electromagnetic nanocommunications in the terahertz band Antennas and Propagation (EuCAP), 2010 Proceedings of the Fourth European Conference on (IEEE) pp 1-5

[156] Hanson G 2008 Antennas and Propagation Magazine, IEEE 50 66-77

[157] Shuba M, Slepyan G Y, Maksimenko S, Thomsen C and Lakhtakia A 2009 Phys. Rev. B 79 155403

[158] Berres J A and Hanson G W 2011 IEEE Trans. Antennas. Propag. 59 3098-3103

[159] Hao J and Hanson G W 2006 Phys. Rev. B 74035119 
[160] Shuba M V, Maksimenko S A and Lakhtakia A 2007 Phys. Rev. B 76155407

[161] Wang Y, Wu Q, Shi W, He X, Sun X and Gui T 2008 International Journal of Infrared and Millimeter Waves 29 35-42

[162] Huang Y, Yin W Y and Liu Q H 2008 IEEE Trans. Nanotechnol. 7 331-337

[163] Maksimenko S, Slepyan G Y, Nemilentsau A and Shuba M 2008 Physica E: Low-dimensional Systems and Nanostructures 40 2360-2364

[164] Sze S M and Ng K K 2006 Physics of semiconductor devices (Wiley. com)

[165] Endoh A, Yamashita Y, Higashiwaki M, Hikosaka K, Mimura T, Hiyamizu S and Matsui T 2000 High $f_{T}$ 50-nm-gate lattice-matched inalas/ingaas hemts Indium Phosphide and Related Materials, 2000. Conference Proceedings. 2000 International Conference on (IEEE) pp 87-90

[166] Javey A, Guo J, Wang Q, Lundstrom M and Dai H 2003 Nature 424 654-657

[167] Baumgardner J E, Pesetski A A, Murduck J M, Przybysz J X, Adam J D and Zhang H 2007 Appl. Phys. Lett. 91 052107-052107

[168] Guo J, Hasan S, Javey A, Bosman G and Lundstrom M 2005 IEEE Trans. Nanotechnol. 4 715-721

[169] Alam K and Lake R 2005 Appl. Phys. Lett. 87 073104-073104

[170] Hasan S, Salahuddin S, Vaidyanathan M and Alam M 2006 IEEE Trans. Nanotechnol. 5 14-22

[171] Pulfrey D L and Chen L 2008 Solid-State Electronics 52 1324-1328

[172] Koswatta S O, Valdes-Garcia A, Steiner M B, Lin Y M and Avouris P 2011 IEEE Trans. Microwave Theory Tech. 59 2739-2750

[173] Li S, Yu Z, Yen S F, Tang W C and Burke P J 2004 Nano Lett. 4 753-756

[174] Wang D, Yu Z, McKernan S and Burke P 2007 IEEE Trans. Nanotechnol. 6 400-403

[175] Chaste J, Lechner L, Morfin P, Feve G, Kontos T, Berroir J M, Glattli D, Happy H, Hakonen P and Plaçais B 2008 Nano Lett. 8 525-528

[176] Le Louarn A, Kapche F, Bethoux J M, Happy H, Dambrine G, Derycke V, Chenevier P, Izard N, Goffman M and Bourgoin J P 2007 Appl. Phys. Lett. 90233108

[177] Yu Z, Rutherglen C and Burke P 2006 Appl. Phys. Lett. 88 233115-233115

[178] Rosenblatt S, Lin H, Sazonova V, Tiwari S and McEuen P L 2005 Appl. Phys. Lett. $87153111-$ 153111

[179] Pesetski A A, Baumgardner J E, Folk E, Przybysz J X, Adam J D and Zhang H 2006 Appl. Phys. Lett. 88 113103-113103

[180] Nougaret L, Dambrine G, Lepilliet S, Happy H, Chimot N, Derycke V and Bourgoin J P 2010 Appl. Phys. Lett. 96 042109-042109

[181] Cobas E D, Anlage S M and Fuhrer M S 2011 IEEE Trans. Microwave Theory Tech. 59 2726-2732

[182] Kocabas C, Kim H s, Banks T, Rogers J A, Pesetski A A, Baumgardner J E, Krishnaswamy S and Zhang H 2008 Proceedings of the National Academy of Sciences 105 1405-1409

[183] Kocabas C, Dunham S, Cao Q, Cimino K, Ho X, Kim H S, Dawson D, Payne J, Stuenkel M, Zhang H et al. 2009 Nano Lett. 9 1937-1943

[184] Steiner M, Engel M, Lin Y M, Wu Y, Jenkins K, Farmer D B, Humes J J, Yoder N L, Seo J W T, Green A A et al. 2012 Appl. Phys. Lett. 101 053123-053123

[185] Nougaret L, Happy H, Dambrine G, Derycke V, Bourgoin J P, Green A and Hersam M 2009 Appl. Phys. Lett. 94 243505-243505

[186] Wang C, Badmaev A, Jooyaie A, Bao M, Wang K L, Galatsis K and Zhou C 2011 ACS nano 5 $4169-4176$

[187] Hu L, Hecht D and Grüner G 2004 Nano Lett. 4 2513-2517

[188] Kocabas C, Pimparkar N, Yesilyurt O, Kang S, Alam M A and Rogers J 2007 Nano Lett. 7 1195-1202

[189] Kang S J, Kocabas C, Ozel T, Shim M, Pimparkar N, Alam M A, Rotkin S V and Rogers J A 2007 Nature Nanotechnol. 2 230-236

[190] Engel M, Small J P, Steiner M, Freitag M, Green A A, Hersam M C and Avouris P 2008 Acs Nano 2 2445-2452

[191] Chimot N, Derycke V, Goffman M F, Bourgoin J P, Happy H and Dambrine G 2007 Appl. Phys. 


\section{Lett. 91 153111-153111}

[192] Che Y, Lin Y C, Kim P and Zhou C 2013 ACS Nano 7 4343-4350

[193] Shon N H and Ando T 1998 J. Phys. Soc. Jpn. 67 2421-2429

[194] Zheng Y and Ando T 2002 Phys. Rev. B 65245420

[195] Peres N M R, Guinea F and Castro Neto A H 2006 Phys. Rev. B 73(12) 125411

[196] Gusynin V P and Sharapov S G 2006 Phys. Rev. B 73(24) 245411

[197] Gusynin V P, Sharapov S G and Carbotte J P 2006 Phys. Rev. Lett. 96(25) 256802

[198] Gusynin V P, Sharapov S G and Carbotte J P 2007 Phys. Rev. Lett. 98(15) 157402

[199] Ryu S, Mudry C, Furusaki A and Ludwig A W W 2007 Phys. Rev. B 75(20) 205344

[200] Abergel D S L and Fal'ko V I 2007 Phys. Rev. B 75(15) 155430

[201] Mishchenko E G 2007 Phys. Rev. Lett. 98(21) 216801

[202] Gusynin V P, Sharapov S G and Carbotte J P 2007 Phys. Rev. B 75(16) 165407

[203] Falkovsky L A and Varlamov A A 2007 Eur. Phys. J. B. 56 281-284

[204] Falkovsky L A and Pershoguba S S 2007 Phys. Rev. B 76(15) 153410

[205] Sheehy D E and Schmalian J 2007 Phys. Rev. Lett. 99(22) 226803

[206] Herbut I F, Juričić V and Vafek O 2008 Phys. Rev. Lett. 100(4) 046403

[207] Koshino M and Ando T 2008 Phys. Rev. B 77(11) 115313

[208] Mishchenko E G 2008 Europhys. Lett. 8317005

[209] Lewkowicz M and Rosenstein B 2009 Phys. Rev. Lett. 102(10) 106802

[210] Ingenhoven P, Bernád J Z, Zülicke U and Egger R 2010 Phys. Rev. B 81(3) 035421

[211] Juričić V, Vafek O and Herbut I F 2010 Phys. Rev. B 82(23) 235402

[212] Vasko F T, Mitin V V, Ryzhii V and Otsuji T 2012 Phys. Rev. B 86235424

[213] Li Z Q, Henriksen E A, Jiang Z, Hao Z, Martin M C, Kim P, Stormer H L and Basov D N 2008 Nature Phys. 4 532-535

[214] Dawlaty J M, Shivaraman S, Strait J, George P, Chandrashekhar M, Rana F, Spencer M G, Veksler D and Chen Y 2008 Appl. Phys. Lett. 93131905

[215] Choi H, Borondics F, Siegel D A, Zhou S Y, Martin M C, Lanzara A and Kaindl R A 2009 Appl. Phys. Lett. 94172102 (pages 3)

[216] Horng J, Chen C F, Geng B, Girit C, Zhang Y, Hao Z, Bechtel H A, Martin M, Zettl A, Crommie M F, Shen Y R and Wang F 2011 Phys. Rev. B 83(16) 165113

[217] Liu W, Aguilar R V, Hao Y, Ruoff R S and Armitage N P 2011 J. Appl. Phys. 110083510 (pages 5)

[218] Yan H, Xia F, Zhu W, Freitag M, Dimitrakopoulos C, Bol A A, Tulevski G and Avouris P 2011 ACS Nano 5 9854-9860

[219] Ren L, Zhang Q, Yao J, Sun Z, Kaneko R, Yan Z, Nanot S, Jin Z, Kawayama I, Tonouchi M, Tour J M and Kono J 2012 Nano Lett. 12 3711-3715

[220] Maeng I, Lim S, Chae S J, Lee Y H, Choi H and Son J H 2012 Nano Lett. 12 551-555

[221] Sensale-Rodriguez B, Yan R, Kelly M M, Fang T, Tahy K, Hwang W S, Jena D, Liu L and Xing H G 2012 Nature Commun. 3780

[222] Talanov V V, Barga C D, Wickey L, Kalichava I, Gonzales E, Shaner E A, Gin A V and Kalugin N G 2010 ACS Nano 4 3831-3838

[223] Buron J D, Petersen D H, Bggild P, Cooke D G, Hilke M, Sun J, Whiteway E, Nielsen P F, Hansen O, Yurgens A and Jepsen P U 2012 Nano Lett. 12 5074-5081

[224] Sadowski M L, Martinez G, Potemski M, Berger C and de Heer W A 2006 Phys. Rev. Lett. 97(26) 266405

[225] Jiang Z, Henriksen E A, Tung L C, Wang Y J, Schwartz M E, Han M Y, Kim P and Stormer H L 2007 Phys. Rev. Lett. 98(19) 197403

[226] Deacon R S, Chuang K C, Nicholas R J, Novoselov K S and Geim A K 2007 Phys. Rev. B 76(8) 081406

[227] Henriksen E A, Jiang Z, Tung L C, Schwartz M E, Takita M, Wang Y J, Kim P and Stormer H L 2008 Phys. Rev. Lett. 100087403 
[228] Orlita M, Faugeras C, Plochocka P, Neugebauer P, Martinez G, Maude D K, Barra A L, Sprinkle M, Berger C, de Heer W A and Potemski M 2008 Phys. Rev. Lett. 101267601

[229] Neugebauer P, Orlita M, Faugeras C, Barra A L and Potemski M 2009 Phys. Rev. Lett. 103 136403

[230] Henriksen E A, Cadden-Zimansky P, Jiang Z, Li Z Q, Tung L C, Schwartz M E, Takita M, Wang Y J, Kim P and Stormer H L 2010 Phys. Rev. Lett. 104067404

[231] Crassee I, Levallois J, Walter A L, Ostler M, Rotenberg A B E, Seyller T, van der Marel D and Kuzmenko A B 2011 Nature Phys. 7 48-51

[232] Witowski A M, Orlita M, Stẹpniewski R, Wysmołek A, Baranowski J M, Strupiński W, Faugeras C, Martinez G and Potemski M 2010 Phys. Rev. B 82(16) 165305

[233] Orlita M, Faugeras C, Grill R, Wysmolek A, Strupinski W, Berger C, de Heer W A, Martinez G and Potemski M 2011 Phys. Rev. Lett. 107216603

[234] Crassee I, Levallois J, van der Marel D, Walter A L, Seyller T and Kuzmenko A B 2011 Phys. Rev. B 84035103

[235] Booshehri L G, Mielke C H, Rickel D G, Crooker S A, Zhang Q, Ren L, Hároz E H, Rustagi A, Stanton C J, Jin Z, Sun Z, Yan Z, Tour J M and Kono J 2012 Phys. Rev. B 85205407

[236] Satou A, Vasko F T and Ryzhii V 2008 Phys. Rev. B 78115431

[237] Dubinov A A, Aleshkin V Y, Ryzhii M, Otsuji T and Ryzhii V 2009 Appl. Phys. Exp. 2092301

[238] Satou A, Otsuji T and Ryzhii V 2011 Jpn. J. Appl. Phys. 50070116

[239] Satou A, Ryzhii V, Kurita Y and Otsuji T 2013 J. Appl. Phys. 113 143108-143108

[240] Rana F and Ahmad F R 2007 arXiv preprint arXiv:0704.0607

[241] Rana F 2008 IEEE Trans. Nanotech. 7 91-99

[242] Ryzhii V, Ryzhii M, Mitin V and Shur M S 2009 Appl. Phys. Exp. 2034503

[243] Ryzhii V, Satou A, Ryzhii M, Otsuji T and Shur M S 2008 J. Phys.: Cond. Matt. 20384207

[244] Dubinov A A, Aleshkin V Y, Mitin V, Otsuji T and Ryzhii V 2011 J. Phys.: Condens. Matter 23145302

[245] Popov V V, Polischuk O V, Davoyan A R, Ryzhii V, Otsuji T and Shur M S 2012 Phys. Rev. B 86195437

[246] Ryzhii V, Ryzhii M, Satou A, Otsuji T, Dubinov A and Aleshkin V Y 2009 J. Appl. Phys. 106 084507-084507

[247] Ryzhii V, Dubinov A A, Otsuji T, Mitin V and Shur M S 2010 J. Appl. Phys. 107054505

[248] Ryzhii M and Ryzhii V 2007 Jpn. J. Appl. Phys. 46 L151-L153

[249] Ryzhii V, Ryzhii M, Mitin V and Otsuji T 2011 J. Appl. Phys. 110 094503-094503

[250] Otsuji T, Tombet S B, Satou A, Fukidome H, Suemitsu M, Sano E, Popov V, Ryzhii M and Ryzhii V 2012 J. Phys. D: Appl. Phys. 45303001

[251] Ryzhii V, Semenikhin I, Ryzhii M, Svintsov D, Vyurkov V, Satou A and Otsuji T 2013 J. Appl. Phys. 113244505

[252] Mikhailov S A 2013 Phys. Rev. B 87115405

[253] Freitag M, Chiu H Y, Steiner M, Perebeinos V and Avouris P 2010 Nature Nano. 5 497-501

[254] Ramakrishnan G, Chakkittakandy R and Planken P C M 2009 Opt. Exp. $1716092-16099$

[255] Sun D, Wu Z K, Divin C, Li X, Berger C, de Heer W A, First P N and Norris T B 2008 Phys. Rev. Lett. 101157402

[256] Dawlaty J M, Shivaraman S, Chandrashekhar M, Rana F and Spencer M G 2008 Appl. Phys. Lett. 92042116

[257] Newson R W, Dean J, Schmidt B and van Driel H M 2009 Opt. Express 17 2326-2333

[258] Breusing M, Ropers C and Elsaesser T 2009 Phys. Rev. Lett. 102086809

[259] Plochocka P, Kossacki P, Golnik A, Kazimierczuk T, Berger C, de Heer W A and Potemski M 2009 Phys. Rev. B 80245415

[260] Wang H, Strait J H, George P A, Shivaraman S, Shields V B, Chandrashekhar M, Hwang J, Rana F, Spencer M G, Ruiz-Vargas C S et al. 2010 Appl. Phys. Lett. 96 081917-081917

[261] Lui C H, Mak K F, Shan J and Heinz T F 2010 Phys. Rev. Lett. 105127404 
[262] Obraztsov P A, Rybin M G, Tyurnina A V, Garnov S V, Obraztsova E D, Obraztsov A N and Svirko Y P 2011 Nano Lett. 11 1540-1545

[263] Hale P J, Hornett S M, Moger J, Horsell D W and Hendry E 2011 Phys. Rev. B 83121404

[264] Breusing M, Kuehn S, Winzer T, Malić E, Milde F, Severin N, Rabe J, Ropers C, Knorr A and Elsaesser T 2011 Phys. Rev. B 83153410

[265] Sun D, Divin C, Berger C, de Heer W A, First P N and Norris T B 2011 Phys. Status Solidi C 8 1194-1197

[266] Brida D, Tomadin A, Manzoni C, Kim Y J, Lombardo A, Milana S, Nair R R, Novoselov K S, Ferrari A C, Cerullo G and Polini M 2013 Nature Commun. 4

[267] George P A, Strait J, Dawlaty J, Shivaraman S, Chandrashekhar M, Rana F and Spencer M G 2008 Nano Lett. 8 4248-4251

[268] Karasawa H, Komori T, Watanabe T, Satou A, Fukidome H, Suemitsu M, Ryzhii V and Otsuji T 2011 Journal of Infrared, Millimeter, and Terahertz Waves 32 655-665

[269] Otsuji T, Tombet S A B, Chan S, Satou A and Ryzhii V 2011 Terahertz light amplification by stimulated emission of radiation from optically pumped graphene SPIE Defense, Security, and Sensing (International Society for Optics and Photonics) pp 802304-802304

[270] Strait J H, Wang H, Shivaraman S, Shields V, Spencer M and Rana F 2011 Nano Lett. 11 $4902-4906$

[271] Boubanga-Tombet S, Chan S, Watanabe T, Satou A, Ryzhii V and Otsuji T 2012 Phys. Rev. B 85035443

[272] Docherty C J, Lin C T, Joyce H J, Nicholas R J, Herz L M, Li L J and Johnston M B 2012 Nature Commun. 31228

[273] Tielrooij K J, Song J C W, Jensen S A, Centeno A, Pesquera A, Zurutuza E A, Bonn M, Levitov L S and Koppens F H L 2013 Nature Phys. 9 248-252

[274] Prechtel L, Song L, Schuh D, Ajayan P, Wegscheider W and Holleitner A W 2012 Nature Commun. 3646

[275] Tani S, Blanchard F and Tanaka K 2012 Phys. Rev. Lett. 109166603

[276] Li T, Luo L, Hupalo M, Zhang J, Tringides M C, Schmalian J and Wang J 2012 Phys. Rev. Lett. 108167401

[277] Winnerl S, Orlita M, Plochocka P, Kossacki P, Potemski M, Winzer T, Malić E, Knorr A, Sprinkle M, Berger C, de Heer W A, Schneider H and Helm M 2011 Phys. Rev. Lett. 107237401

[278] Jnawali G, Rao Y, Yan H and Heinz T F 2013 Nano Lett. 13 524-530

[279] Kim J, Lim S C, Chae S J, Maeng I, Choi Y, Cha S, Lee Y H and Choi H 2013 Sci. Rep. 3

[280] Jiang C, Shalygin V, Panevin V Y, Danilov S N, Glazov M, Yakimova R, Lara-Avila S, Kubatkin S and Ganichev S 2011 Phys. Rev. B 84125429

[281] Rana F 2007 Phys. Rev. B $\mathbf{7 6} 155431$

[282] Butscher S, Milde F, Hirtschulz M, Malic E and Knorr A 2007 Appl. Phys. Lett. 91 203103-203103

[283] Bistritzer R and MacDonald A H 2009 Phys. Rev. Lett. 102206410

[284] Romanets P N and Vasko F T 2010 Phys. Rev. B 81085421

[285] Winzer T, Knorr A and E M 2010 Nano Lett. 10 4839-4843

[286] Kim R, Perebeinos V and Avouris P 2011 Phy. Rev. B 84075449

[287] Malić E, Winzer T, Bobkin E and Knorr A 2011 Phy. Rev. B 84205406

[288] Sun B Y, Zhou Y and Wu M W 2012 Phys. Rev. B 85125413

[289] Winzer T and Malić E 2012 Phys. Rev. B 85241404

[290] Winzer T, Malić E and Knorr A 2013 Phys. Rev. B 87165413

[291] Sun B Y and Wu M W 2013 New J. Phys. 15083038

[292] Foster M S and Aleiner I L 2009 Phys. Rev. B 79085415

[293] Mikhailov S A and Ziegler K 2008 J. Phys.: Cond. Matt. 20384204

[294] Glazov M M and Ganichev S D 2014 Phys. Rep. 535 101-138

[295] Wright A, Xu X, Cao J and Zhang C 2009 Appl. Phys. Lett. 95072101

[296] Ishikawa K L 2010 Phys. Rev. B 82201402 
[297] Paul M, Chang Y, Thompson Z, Stickel A, Wardini J, Choi H, Minot E, Norris T and Lee Y S 2013 New J. Phys. 15085019

[298] Olbrich P, Drexler C, Golub L, Danilov S, Shalygin V, Yakimova R, Lara-Avila S, Kubatkin S, Redlich B, Huber R et al. 2013 Phys. Rev. B 88245425

[299] Calvo H L, Pastawski H M, Roche S and Foa Torres L E F 2011 Appl. Phys. Lett. 98232103

[300] Syzranov S V, Fistul M V and Efetov K B 2008 Phys. Rev. B 78045407

[301] López-Rodríguez F J and Naumis G G 2008 Phys. Rev. B 78 201406(R)

[302] Oka T and Aoki H 2009 Phys. Rev. B 79 081406(R)

[303] Kibis O 2010 Phys. Rev. B 81165433

[304] Kibis O, Kyriienko O and Shelykh I 2011 Phys. Rev. B 84195413

[305] Gumbs G, Roslyak O, Huang D and Balassis A 2011 J. Mod. Opt. 58 1990-1996

[306] Zhou Y and Wu M W 2011 Phys. Rev. B 83245436

[307] Perez-Piskunow P M, Usaj G, Balseiro C A and Foa Torres L E F 2014 Phys. Rev. B 89(12) 121401

[308] Calvo H L, Perez-Piskunow P M, Roche S and Foa Torres L E 2012 Appl. Phys. Lett. 101253506

[309] Mikhailov S A 2009 Phys. Rev. B 79(24) 241309(R)

[310] Yao X and Belyanin A 2012 Phys. Rev. Lett. 108255503

[311] Kuzmenko A, Van Heumen E, Carbone F and Van Der Marel D 2008 Phys. Rev. Lett. 100117401

[312] Thongrattanasiri S, Koppens F H and de Abajo F J G 2012 Phys. Rev. Lett. 108047401

[313] Fang Z, Liu Z, Wang Y, Ajayan P M, Nordlander P and Halas N J 2012 Nano Lett. 12 3808-3813

[314] Sensale-Rodriguez B, Yan R, Rafique S, Zhu M, Li W, Liang X, Gundlach D, Protasenko V, Kelly M M, Jena D, Liu L and Xing H G 2012 Nano Lett. 12 4518-4522

[315] Strikha M V and Vasko F T 2010 Phys. Rev. B 81115413

[316] Strikha M V and Vasko F T 2011 J. Appl. Phys. 110083106

[317] Vafek O 2006 Phys. Rev. Lett. 97266406

[318] Apalkov V, Wang X F and Chakraborty T 2007 Int. J. Mod Phys B 21 1165-1179

[319] Wang X F and Chakraborty T 2007 Phys. Rev. B 75033408

[320] Hwang E H and Das Sarma S 2007 Phys. Rev. B 75205418

[321] Ryzhii V, Satou A and Otsuji T 2007 J. Appl. Phys. 101024509

[322] Mikhailov S A and Ziegler K 2007 Phys. Rev. Lett. 99(1) 016803

[323] Hanson G W 2008 J. Appl. Phys. $103064302-064302$

[324] Jablan M, Buljan H and Soljačić M 2009 Phys. Rev. B 80245435

[325] Nikitin A Y, Guinea F, Garcia-Vidal F J and Martin-Moreno L 2011 Phys. Rev. B 84161407

[326] Koppens F H, Chang D E and Garcia de Abajo F J 2011 Nano Lett. 11 3370-3377

[327] Huidobro P A, Nikitin A Y, González-Ballestero C, Martín-Moreno L and García-Vidal F J 2012 Phys. Rev. B $\mathbf{8 5} 155438$

[328] Peres N M R, Ferreira A, Bludov Y V and Vasilevskiy M I 2012 J. Phys.: Condens. Matter 24 245303

[329] Gómez-Díaz J and Perruisseau-Carrier J 2012 J. Appl. Phys. 112 124906-124906

[330] Davoyan A R, Popov V V and Nikitov S A 2012 Phys. Rev. Lett. 108127401

[331] Bludov Y V, Peres N M R and Vasilevskiy M I 2012 Phys. Rev. B 85245409

[332] Slipchenko T, Nesterov M, Martin-Moreno L and Nikitin A Y 2013 arXiv preprint arXiv:130\%.0310

[333] Garcia-Pomar J L N A Y and L M M 2013 ACS Nano 74988

[334] Bludov Y V, Ferreira A, Peres N M R and Vasilevskiy M I 2013 International Journal of Modern Physics B $\mathbf{2 7}$

[335] Yao X, Tokman M and Belyanin A 2013 arXiv preprint arXiv:1308.2005

[336] Ju L, Geng B, Horng J, Girit C, Martin M, Hao Z, Bechtel H A, Liang X, Zettl A, Shen Y R and Wang F 2011 Nature Nano. 6 630-634

[337] Chen J, Badioli M, Alonso-González P, Thongrattanasiri S, Huth F, Osmond J, Spasenović M, Centeno A, Pesquera A, Godignon P et al. 2012 Nature 487 77-81 
[338] Fei Z, Rodin A, Andreev G, Bao W, McLeod A, Wagner M, Zhang L, Zhao Z, Thiemens M, Dominguez G et al. 2012 Nature 487 82-85

[339] Yan H, Low T, Zhu W, Wu Y, Freitag M, Li X, Guinea F, Avouris P and Xia F 2013 Nature Photon. 7 394-399

[340] Petković I, Williams F I B, Bennaceur K, Portier F, Roche P and Glattli D C 2013 Phys. Rev. Lett. 110016801

[341] Crassee I, Orlita M, Potemski M, Walter A L, Ostler M, Seyller T, Gaponenko I, Chen J and Kuzmenko A B 2012 Nano Lett. 12 2470-2474

[342] Yan H, Li X, Chandra B, Tulevski G, Wu Y, Freitag M, Zhu W, Avouris P and Xia F 2012 Nature Nano. 7 330-334

[343] Lee S H, Choi M, Kim T T, Lee S, Liu M, Yin X, Choi H K, Lee S S, Choi C G, Choi S Y, Zhang $\mathrm{X}$ and Min B 2012 Nature Mater. 11 936-941

[344] Gao W, Shu J, Reichel K, Nickel D V, He X, Shi G, Vajtai R, Ajayan P M, Kono J, Mittleman D M and Xu Q 2014 Nano Lett. 0 null

[345] Bludov Y V, Vasilevskiy M I and Peres N M R 2010 Europhys. Lett. 9268001

[346] Echtermeyer T J, Britnell L, Jasnos P K, Lombardo A, Gorbachev R V, Grigorenko A N, Geim A K, Ferrari A C and Novoselov K S 2011 Nature Communications 2458

[347] Zhan T R, Zhao F Y, Hu X H, Liu X H and Zi J 2012 Phys. Rev. B 86165416

[348] Gao W, Shu J, Qiu C and Xu Q 2012 ACS nano 6 7806-7813

[349] Peres N M R, Bludov Y V, Ferreira A and Vasilevskiy M I 2013 J. Phys.: Condens. Matter 25 125303

[350] Nikitin A Y, Guinea F, Garcia-Vidal F J and Martin-Moreno L 2012 Phys. Rev. B 85081405

[351] Mishchenko E G, Shytov A V and Silvestrov P G 2010 Phys. Rev. Lett 104156806

[352] Thongrattanasiri S, Silveiro I and Javier García de Abajo F 2012 Appl. Phys. Lett. 100 201105201105

[353] Popov V V, Bagaeva T Y, Otsuji T and Ryzhii V 2010 Phys. Rev. B 8173404

[354] Vakil A and Engheta N 2011 Science 332 1291-1294

[355] Chamanara N, Sounas D and Caloz C 2013 Opt. Express 21 11248-11256

[356] Christensen J, Manjavacas A, Thongrattanasiri S, Koppens F H L and García de Abajo F J 2011 ACS nano 6 431-440

[357] Gómez-Díaz J S and Perruisseau-Carrier J 2013 Opt. Express 21 15490-15504

[358] Amin M, Farhat M and Bağcı H 2013 Sci. Rep. 3

[359] Liu M, Yin X, Ulin-Avila E, Geng B, Zentgraf T, Ju L, Wang F and Zhang X 2011 Nature 474 64-67

[360] Chen P, Argyropoulos C and Alù A 2013 IEEE Trans. Antennas Propag. 61 1528-1537

[361] Serrano D C, Gómez-Díaz J S, Perruisseau-Carrier J and Melcon A A 2013 arXiv preprint arXiv:1304.6320

[362] Fallahi A and Perruisseau-Carrier J 2012 Phys. Rev. B 86195408

[363] Carrasco E, Tamagnone M and Perruisseau-Carrier J 2013 Appl. Phys. Lett. 102 104103-104103

[364] Dragoman M, Muller A A, Dragoman D, Coccetti F and Plana R 2010 J. Appl. Phys. 107 104313-104313

[365] Huang Y, Wu L S, Tang M and Mao J 2012 IEEE Trans. on Nanotechnol. 11 836-842

[366] Martí I L, Kremers C, Cabellos-Aparicio A, Jornet J M, Alarcón E and Chigrin D N 2011 Scattering of terahertz radiation on a graphene-based nano-antenna vol 1398 p 144

[367] Llatser I, Kremers C, Cabellos-Aparicio A, Jornet J M, Alarcón E and Chigrin D N 2012 Photon Nanostruct: Fundam. Appl $10353-358$

[368] Tamagnone M, Gómez-Díaz J S, Mosig J R and Perruisseau-Carrier J 2012 Appl. Phys. Lett. 101 214102

[369] Filter R, Farhat M, Steglich M, Alaee R, Rockstuhl C and Lederer F 2013 Opt. Express 21 $3737-3745$

[370] Esquius-Morote M, Gómez-Díaz J S and Perruisseau-Carrier J 2013 arXiv preprint 
arXiv:1305.477/4

[371] Ryzhii V, Ryabova N, Ryzhii M, Baryshnikov N, Karasik V, Mitin V and Otsuji T 2012 OptoElectronics Rev. 20 15-25

[372] Ryzhii V, Ryzhii M, Mitin V and Otsuji T 2010 J. Appl. Phys. $107054512-054512$

[373] Ryzhii V, Ryzhii M, Ryabova N, Mitin V and Otsuji T 2011 J. Infrared Phys. Technol. 54 302-305

[374] Ryzhii M, Otsuji T, Mitin V and Ryzhii V 2011 Jpn. J. Appl. Phys 50 070117-1

[375] Ryzhii V and Ryzhii M 2009 Phys. Rev. B 79245311

[376] Ryzhii V, Mitin V, Ryzhii M, Ryabova N and Otsuji T 2008 Appl. Phys. Exp. 1063002

[377] Ryzhii V, Otsuji T, Ryabova N, Ryzhii M, Mitin V and Karasik V 2012 Infrared Physics 8 Technology

[378] Ryzhii V, Otsuji T, Ryzhii M and Shur M 2012 J. Phys. D: Appl. Phys. 45302001

[379] Ryzhii V, Satou A, Otsuji T and Shur M S 2008 J. Appl. Phys. 103014504

[380] Xia F, Mueller T, Lin Y m, Valdes-Garcia A and Avouris P 2009 Nature Nanotechnol. 4 839-843

[381] Mueller T X F and Avouris P 2010 Nature Photon. 4297

[382] Vicarelli L, Vitiello M S, Coquillat D, Lombardo A, Ferrari A C, Knap W, Polini M, Pellegrini V and Tredicucci A 2012 Nature Mater. 11 865-871

[383] Cai X, Sushkov A B, Suess R J, Jenkins G S, Yan J, Murphy T E, Drew H D and Fuhrer M S 2013 arXiv preprint arXiv:1305.3297

[384] Lee E J H, Balasubramanian K, Weitz R T, Burghard M and Kern K 2008 Nature Nano. 3 486-490

[385] Mueller T, Xia F, Freitag M, Tsang J and Avouris P 2009 Phys. Rev. B 79245430

[386] Xia F, Mueller T, Golizadeh-Mojarad R, Freitag M, Lin Y m, Tsang J, Perebeinos V and Avouris P 2009 Nano Lett. 9 1039-1044

[387] Fong K and Schwab K 2012 Phys. Rev. X 2031006

[388] McKitterick C B, Prober D E and Karasik B S 2013 J. Appl. Phys. $113044512-044512$

[389] McKitterick C B, Vora H, Du X, Karasik B S and Prober D E 2013 arXiv preprint arXiv:1307.5012

[390] Yan J, Kim M, Elle J, Sushkov A, Jenkins G, Milchberg H, Fuhrer M and Drew H 2012 Nature Nanotech. 7 472-478

[391] Kim M H, Yan J, Suess R J, Murphy T E, Fuhrer M S and Drew H D 2013 Phys. Rev. Lett. 110(24) 247402

[392] McCann E and Falko V I 2006 Phys. Rev. Lett. 96086805

[393] Ryzhii V, Otsuji T, Ryzhii M, Ryabova N, Yurchenko S, Mitin V and Shur M 2013 J. Phys. D: Appl. Phys. 46065102

[394] Kalugin N G, Jing L, Bao W, Wickey L, Del Barga C, Ovezmyradov M, Shaner E A and Lau C N 2011 Appl. Phys. Lett. 99 013504-013504

[395] Chitara B, Panchakarla L, Krupanidhi S and Rao C 2011 Adv. Mater. 23 5419-5424

[396] Karch J, Olbrich P, Schmalzbauer M, Zoth C, Brinsteiner C, Fehrenbacher M, Wurstbauer U, Glazov M M, Tarasenko S A, Ivchenko E L, Weiss D, Eroms J, Yakimova R, Lara-Avila S, Kubatkin S and Ganichev S D 2010 Phys. Rev. Lett. 105227402

[397] Karch J, Drexler C, Olbrich P, Fehrenbacher M, Hirmer M, Glazov M M, Tarasenko S A, Ivchenko E L, Birkner B, Eroms J, Weiss D, Yakimova R, Lara-Avila S, Kubatkin S, Ostler M, Seyller T and Ganichev S D 2011 Phys. Rev. Lett. 107276601

[398] Drexler C, Tarasenko S, Olbrich P, Karch J, Hirmer M, Müller F, Gmitra M, Fabian J, Yakimova R, Lara-Avila S et al. 2013 Nat. Nanotechnol. 8 104-107

[399] Mittendorff M, Winnerl S, Kamann J, Eroms J, Weiss D, Schneider H and Helm M 2013 Appl. Phys. Lett. 103021113

[400] Kazarinov R F and Suris R A 1971 Sov. Phys. Semiconductors 5 797-800

[401] Faist J, Capasso F, Sivco D L, Sirtori C, Hutchinson A L and Cho A Y 1994 Science 264 553-556 